\title{
Statistics of velocity and temperature fluctuations in two-dimensional Rayleigh-Bénard convection
}

\author{
Yang Zhang, ${ }^{1}$ Yong-Xiang Huang, ${ }^{2}$ Nan Jiang, ${ }^{3}$ Yu-Lu Liu, ${ }^{1,4}$ Zhi-Ming Lu, ${ }^{1}$ Xiang Qiu, ${ }^{4}$ and Quan Zhou ${ }^{1, *}$ \\ ${ }^{1}$ Shanghai Institute of Applied Mathematics and Mechanics and Shanghai Key Laboratory of Mechanics in Energy Engineering, \\ Shanghai University, Shanghai 200072, China \\ ${ }^{2}$ State Key Laboratory of Marine Environmental Science, Xiamen University, Xiamen 361102, China \\ ${ }^{3}$ Department of Mechanics, Tianjin University, Tianjin 300072, China \\ ${ }^{4}$ School of Science, Shanghai Institute of Technology, Shanghai 200235, China \\ (Received 25 March 2017; revised manuscript received 14 June 2017; published 22 August 2017)
}

\begin{abstract}
We investigate fluctuations of the velocity and temperature fields in two-dimensional (2D) RayleighBénard (RB) convection by means of direct numerical simulations (DNS) over the Rayleigh number range $10^{6} \leqslant \operatorname{Ra} \leqslant 10^{10}$ and for a fixed Prandtl number $\operatorname{Pr}=5.3$ and aspect ratio $\Gamma=1$. Our results show that there exists a counter-gradient turbulent transport of energy from fluctuations to the mean flow both locally and globally, implying that the Reynolds stress is one of the driving mechanisms of the large-scale circulation in 2D turbulent $\mathrm{RB}$ convection besides the buoyancy of thermal plumes. We also find that the viscous boundary layer (BL) thicknesses near the horizontal conducting plates and near the vertical sidewalls, $\delta_{u}$ and $\delta_{v}$, are almost the same for a given $\mathrm{Ra}$, and they scale with the Rayleigh and Reynolds numbers as $\sim \mathrm{Ra}^{-0.26 \pm 0.03}$ and $\sim \mathrm{Re}^{-0.43 \pm 0.04}$. Furthermore, the thermal BL thickness $\delta_{\theta}$ defined based on the root-mean-square (rms) temperature profiles is found to agree with Prandtl-Blasius predictions from the scaling point of view. In addition, the probability density functions of turbulent energy $\varepsilon_{u^{\prime}}$ and thermal $\varepsilon_{\theta^{\prime}}$ dissipation rates, calculated, respectively, within the viscous and thermal BLs, are found to be always non-log-normal and obey approximately a Bramwell-Holdsworth-Pinton distribution first introduced to characterize rare fluctuations in a confined turbulent flow and critical phenomena.
\end{abstract}

DOI: 10.1103/PhysRevE.96.023105

\section{INTRODUCTION}

Buoyancy-driven fluid flows are ubiquitous in geophysics, astrophysics, and process technology. A paradigmatic example that has been widely used to study this type of flow is the so-called Rayleigh-Bénard (RB) convection, i.e., a fluid layer heated uniformly from below and cooled from above [1-4]. In a closed sample, the RB system is determined by three control parameters: the Rayleigh number $\mathrm{Ra}=\alpha g \Delta H^{3} /(\nu \kappa)$, the Prandtl number $\operatorname{Pr}=v / \kappa$, and the aspect ratio $\Gamma=D / H$. Here $\alpha$ is the thermal expansion coefficient, $g$ is the acceleration of gravity, $\Delta$ is the sustained temperature difference between the bottom and top plates of the sample, $v$ is the kinematic viscosity, $\kappa$ is the thermal diffusivity, and $H$ and $D$ are the height and width of the sample, respectively. The response of the system is characterized by the global heat and momentum transports, which are expressed as the nondimensional Nusselt number $\mathrm{Nu}$ and Reynolds number Re, respectively. The scalings of the Nusselt and Reynolds numbers have been extensively studied over the past few years, and we refer interested readers to the recent papers by Ahlers et al. [1] and by Chillà and Schumacher [3] for more details.

A prevalent structure in turbulent RB convection is largescale circulation (LSC, also denoted as the "wind" of turbulence). Krishnamurti and Howard [5] first observed the generation of this large-scale flow, and they argued that the mean flow is apparently maintained against viscous dissipation by the Reynolds stress divergence of the tilted plume motions [5]. This statement motivated researchers to experimentally investigate the turbulent energy production

\footnotetext{
*Author to whom all correspondence should be addressed: qzhou@shu.edu.cn
}

fields in an RB system by using the particle image velocimetry (PIV) technique, and their results showed that the mean wind is driven by the buoyancy of plumes [6-8]. However, due to the restriction of the PIV technique, they could not get the full information on the velocity field.

The boundary layers (BLs) in the vicinity of the conducting plates contribute to the main resistance for the heat transfer, thus they deserve special attention. The viscous and thermal BL thicknesses in RB convection can be defined in several ways: the most popular style is to define them through the time-averaged velocity and temperature profiles near the plates, i.e., the so-called slope method; another way to define the $\mathrm{BL}$ thicknesses is the one employed by the classical laminar boundary layer theories known as 99\% criterion [1]. Recently, Petschel et al. [9,10] introduced the dissipation layers in RB convection. In addition to the methods illustrated above, BL thicknesses can also be determined from the profiles of root-mean-square (rms) values of velocity and temperature fluctuations close to the plates. The properties of this type of BL are used to predict turbulent heat transport and distinguish scaling theories that are based on different assumptions in RB convection [11]. In comparison with those theories, experimental studies have shown some ambiguous observations [12-15]. Additionally, for the Ra dependence of the kinetic BL thickness, Qiu and Xia found that it scales differently near the sidewalls and the bottom plates $[16,17]$.

Velocity and temperature fluctuations play an essential role in the thermal convection system. Once the Rayleigh number is sufficiently high, convection becomes turbulent, the velocity and temperature fields display complex fluctuations both temporally and spatially, and due to buoyancy of the plumes they interplay with each other. The fluctuations of velocity and temperature in turbulent $\mathrm{RB}$ convection have received significant attention from researchers during the past 
couple of years, primarily concentrated on determining the local heat flux [18-20] and the dynamics that drive the cascades of turbulent fluctuations from large to small scales [2,21-23]. In this paper, we want to present the statistical properties of velocity and temperature fluctuations in 2D RB convection by means of direct numerical simulation (DNS), which allow us to get full information on the flow field. Strictly speaking, all convection flows occurring in both natural situations and the laboratory are three-dimensional (3D). Nevertheless, previous investigations have shown that $2 \mathrm{D}$ and $3 \mathrm{D} \mathrm{RB}$ flow share some similarities [24-26], such as the 2D characters of the LSC, the Nu-Ra scaling, the boundary layer profiles, and so forth. Therefore, 2D simulations are conducted to better understand the convection phenomena occurring in the real world. Recently, there has been increasing interest in 2D RB convection [27,28]. For example, it has been used to study the structures of viscous and thermal BLs [29,30], to investigate the flow organization influenced by aspect ratio and velocity boundary conditions [31-33], to compare the differences between 2D and 3D thermal convection [25,26], and to reveal the dynamics of the flow reversals [24,34-36]. Although velocity and temperature fluctuations have been investigated intensively in 3D turbulent RB convection during the past several years [1], to the best of our knowledge a study of velocity and temperature fluctuations in $2 \mathrm{D} \mathrm{RB}$ convection has not been conducted yet.

The remainder of this paper is organized as follows. The governing equations and numerical scheme are briefly introduced in Sec. II. Section III contains the main results of our numerical simulations: Sec. III A contains general features of the flow field; Sec. III B presents the spatial distribution of the turbulent fluctuations; Sec. III C contains the mean profiles of rms velocity and temperature fluctuations and the scalings of BL thicknesses; and in Sec. III D, as a supplement, turbulent dissipation rates in the BLs are investigated by the means of probability density functions (PDFs). We summarize our findings and present the conclusions in Sec. IV.

\section{NUMERICAL METHOD}

We deal with the dimensionless Oberbeck-Boussinesq equations for the incompressible RB problem numerically. The governing equations are as follows:

$$
\begin{gathered}
\frac{\partial \mathbf{u}}{\partial t}+(\mathbf{u} \cdot \nabla) \mathbf{u}=-\nabla p+\sqrt{\frac{\operatorname{Pr}}{\operatorname{Ra}}} \nabla^{2} \mathbf{u}+\theta \mathbf{z}, \\
\nabla \cdot \mathbf{u}=\mathbf{0}, \\
\frac{\partial \theta}{\partial t}+(\mathbf{u} \cdot \nabla) \theta=\sqrt{\frac{1}{\operatorname{PrRa}}} \nabla^{2} \theta,
\end{gathered}
$$

where $\mathbf{u}=u \mathbf{x}+v \mathbf{z}$ ( $\mathbf{x}$ and $\mathbf{z}$ are the horizontal and vertical unit vectors, respectively) is the velocity, $t$ is the time, $p$ is the pressure, and $\theta$ is the temperature. The physical variables length, temperature, velocity, and time are normalized by the height of the cell $H$, the temperature difference $\Delta$, the free-fall velocity $\sqrt{\alpha g \Delta H}$, and the free-fall time $\sqrt{H /(\alpha g \Delta)}$. No-penetration and no-slip velocity boundary conditions are applied to all four solid walls. For temperature $\theta$ the two vertical sidewalls are imposed to be insulated, i.e., no heat
TABLE I. Parameters of the different simulations (columns from left to right): Ra, grid resolutions in horizontal and vertical directions $N_{x} \times N_{z}, \mathrm{Nu}, \mathrm{Re}$, and the averaged time of the simulations $\tau_{0}$ in free-fall time units $\sqrt{H /(\alpha g \Delta)}$.

\begin{tabular}{lcrrrrr}
\hline \hline $\mathrm{Ra}$ & $N_{x} \times N_{z}$ & \multicolumn{1}{c}{$\mathrm{Nu}$} & $\mathrm{Nu}_{\varepsilon_{u}}$ & \multicolumn{1}{c}{$\mathrm{Nu}_{\varepsilon_{\theta}}$} & $\mathrm{Re}$ & $\tau_{0}$ \\
\hline $1 \times 10^{6}$ & $129 \times 129$ & 6.87 & 6.89 & 6.89 & 38 & 7500 \\
$3 \times 10^{6}$ & $193 \times 193$ & 9.33 & 9.35 & 9.34 & 70 & 7500 \\
$1 \times 10^{7}$ & $257 \times 257$ & 13.28 & 13.30 & 13.29 & 156 & 6000 \\
$3 \times 10^{7}$ & $385 \times 385$ & 18.86 & 18.88 & 18.87 & 296 & 4500 \\
$1 \times 10^{8}$ & $513 \times 513$ & 26.21 & 26.22 & 26.22 & 596 & 3000 \\
$3 \times 10^{8}$ & $769 \times 769$ & 35.96 & 36.20 & 35.96 & 1145 & 3000 \\
$1 \times 10^{9}$ & $1025 \times 1025$ & 51.28 & 51.43 & 51.31 & 2269 & 2500 \\
$3 \times 10^{9}$ & $1537 \times 1537$ & 71.57 & 71.54 & 71.71 & 4330 & 1200 \\
$1 \times 10^{10}$ & $2049 \times 2049$ & 105.8 & 106.0 & 105.7 & 9916 & 1000 \\
\hline \hline
\end{tabular}

flux, while the top and bottom plates are held at constant temperatures of -0.5 and +0.5 , respectively.

The numerical method is based on a compact fourth-order finite-difference scheme introduced by Liu et al. [37], and it has been explained by Zhou [38] and Huang and Zhou [39]. Recently, we have employed the same numerical code to investigate the small-scale cascade properties in the $2 \mathrm{D}$ Rayleigh-Taylor system [40-42]. The grid spacing is uniform in both horizontal and vertical directions, and the number of grid points is increased from $129 \times 129$ to $2049 \times 2049$ as Ra increases from $10^{6}$ to $10^{10}$ in the present work. The grid resolution has been chosen to achieve a full resolution of the BLs [43] and to resolve the smallest scales of the problem, i.e., the Kolmogorov scale $\eta_{K}$ and the Batchelor scale $\eta_{B}$. The details about the grid resolution of the present simulations have been given in Table 1 of Ref. [44]: for all simulation cases, the thermal BLs (here the thermal BL thickness is calculated as $\delta_{\text {th }}=\frac{H}{2 \mathrm{Nu}}$ ) are resolved with at least 10 grid points, and the grid spacings $\Delta_{g}<0.21 \eta_{K}$ and $\Delta_{g}<0.49 \eta_{B}$. The fluid is water at $31^{\circ} \mathrm{C}$, corresponding to the Prandtl number $\operatorname{Pr}=5.3$, and the aspect ratio $\Gamma$ of the cell is equal to 1 .

The main parameters of the numerical simulations in this study are summarized in Table I. The integral quantities, i.e., the Nusselt and Reynolds numbers, are calculated as $\mathrm{Nu}=\sqrt{\operatorname{RaPr}}\langle v \theta\rangle_{V, t}+1$ and $\operatorname{Re}=\sqrt{\left\langle\mathbf{u}^{2}\right\rangle_{V, t} \operatorname{Ra} / \operatorname{Pr}}$. Here the notation $\langle\cdot\rangle_{V, t}$ denotes the volume and time average. In a RB convection cell, using the governing equations and the rigid boundary conditions, the two exact relations for the kinetic energy dissipation rate $\left\langle\varepsilon_{u}\right\rangle_{V, t}=v^{3} H^{-4} \operatorname{Pr}^{-2} \operatorname{Ra}(\mathrm{Nu}-1)$ and the thermal energy dissipation rate $\left\langle\varepsilon_{\theta}\right\rangle_{V, t}=\kappa \Delta^{2} H^{-2} \mathrm{Nu}$ can be derived immediately [1-3]. Meanwhile, the nondimensional forms for the two exact expressions are given, respectively, by $\left\langle\tilde{\varepsilon}_{u}\right\rangle_{V, t}=(\mathrm{Nu}-1) / \sqrt{\mathrm{PrRa}}$ and $\left\langle\tilde{\varepsilon}_{\theta}\right\rangle_{V, t}=\mathrm{Nu} / \sqrt{\mathrm{PrRa}}$. The mean heat transfer calculated in $\mathrm{Nu}_{\varepsilon_{u}}=\sqrt{\operatorname{RaPr}}\left\langle\tilde{\varepsilon}_{u}\right\rangle_{V, t}+1$ and $\mathrm{Nu}_{\varepsilon_{\theta}}=\sqrt{\operatorname{RaPr}}\left\langle\tilde{\varepsilon}_{\theta}\right\rangle_{V, t}$ is presented in Table I as well. One sees that for all of the simulations, the differences among $\mathrm{Nu}, \mathrm{Nu}_{\varepsilon_{u}}$, and $\mathrm{Nu}_{\varepsilon_{\theta}}$ are less than $1 \%$, thus guaranteeing the accuracy of our simulations. To further check the accuracy, we compare the response parameters $\mathrm{Nu}$ and $\mathrm{Re}$ with numerical results from the literature. Figure 1 shows a semilogarithmic plot of $\mathrm{Nu} / \mathrm{Ra}^{1 / 3}$ as a function of Ra compared with $3 \mathrm{D}$ simulations. As previously reported, the $\mathrm{Nu}-\mathrm{Ra}$ scalings for the $2 \mathrm{D}$ and 


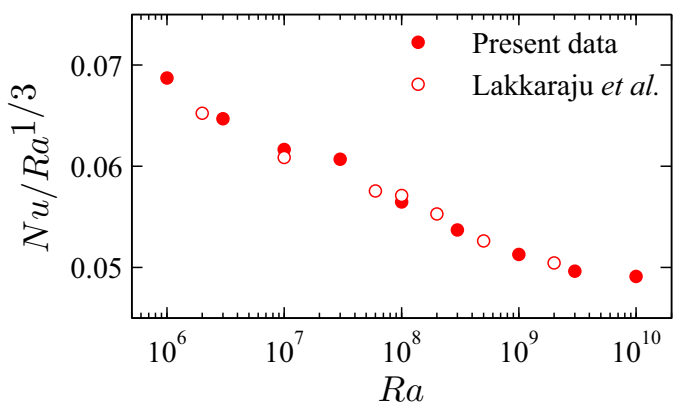

FIG. 1. Ra dependence of the compensated Nusselt number $\mathrm{Nu} / \mathrm{Ra}^{1 / 3}$, the simulation results for $2 \mathrm{D} \mathrm{RB}$ convection are indicated by solid circles, and the 3D numerical results conducted in a cylindrical convection cell by Lakkaraju et al. [20] $(\operatorname{Pr}=5.2)$ are marked with open circles. Note that the 3D data have been multiplied by a factor of 0.79 , which agrees well with the constant 0.78 suggested by van der Poel et al. [26].

3D cases are very similar, differing only by a constant factor in the present parameter range [26]. The global momentum transport scales as $\mathrm{Re} \sim \mathrm{Ra}^{0.60 \pm 0.01}$ (not shown here), which is consistent with the numerical results of $2 \mathrm{D}$ Boussinesq RB convection calculated by Sugiyama et al. [45].

\section{RESULTS AND DISCUSSION}

\section{A. Flow fields}

Although the present study concerns a 2D situation, the dynamics in the system is rather complex. Indeed, the main convection roll exhibits irregular reversals and could reorient its direction due to the energy and size growth of corner-flow rolls [24,34]. The results of Huang and Zhou [39] further revealed that the competition between the corner-flow rolls and the LSC generates countergradient heat transport, which leads to an anomalous $\mathrm{Nu}-\mathrm{Pr}$ relation in the $2 \mathrm{D}$ domain. Typical snapshots of the instantaneous velocity and temperature flow fields for three different Ra spanning four orders of magnitude are plotted in Figs. 2(a)-2(c). They illustrate the general features of the thermal plumes and the LSC in 2D RB convection systems. At the lowest Ra, thermal plumes extend approximately over the entire cell, and they extract thermal energy from the boundary layer and drive the motion of the fluid. From the visualizations (not shown here) corresponding to Fig. 2, it is found that the LSC is not stable at $\mathrm{Ra}=10^{6}$ and the flow reversals occur very frequently. In our simulations, the LSC appears to be stable for $\mathrm{Ra} \gtrsim 3 \times 10^{6}$, roughly the same as the experimental investigations. In those experiments, researchers have found that the large-scale convection roll occurs at $\mathrm{Ra} \gtrsim 2 \times 10^{6}$ and seems to persist even at much higher Rayleigh number by using semiquantitative techniques and quantitative measurements, although the direction of the LSC is occasional, i.e., either clockwise or anticlockwise [6]. At $\mathrm{Ra}=10^{8}$, the LSC has been well-established, rotating with the axis perpendicular to the convection roll's plane, and diagonally orientated in the cell with two counter-rotating rolls in the upper-right and lower-left corners. At $\mathrm{Ra}=10^{10}$, the mushroom-like plumes are well mixed by the enhanced turbulent background, and the LSC becomes more concentrated near the boundary of the cell. To overcome the problem that clockwise and anticlockwise LSC will contribute to zero velocity everywhere in the cell when performing the long time average, we introduce the global angular velocity $\Omega(t)=\langle v /(x-0.5 D)-u /(z-0.5 H)\rangle_{V}$ to
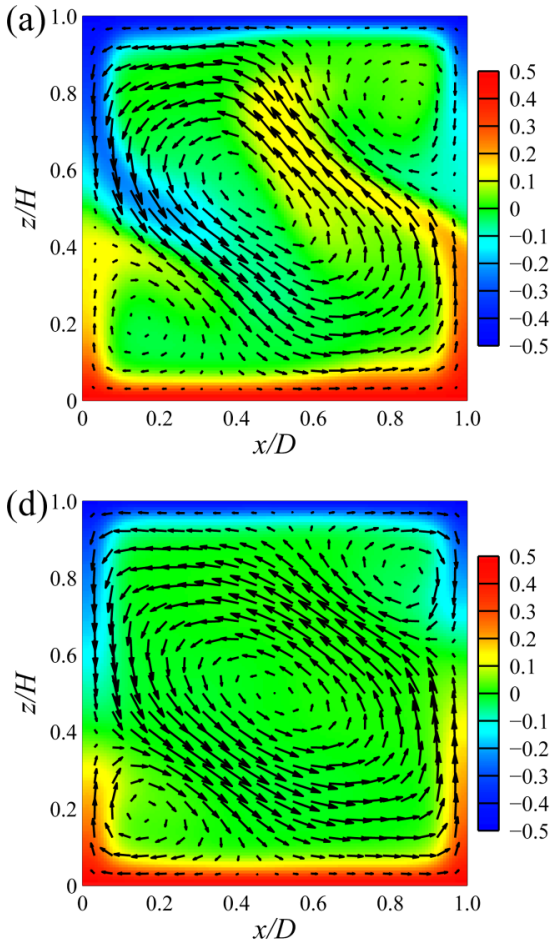
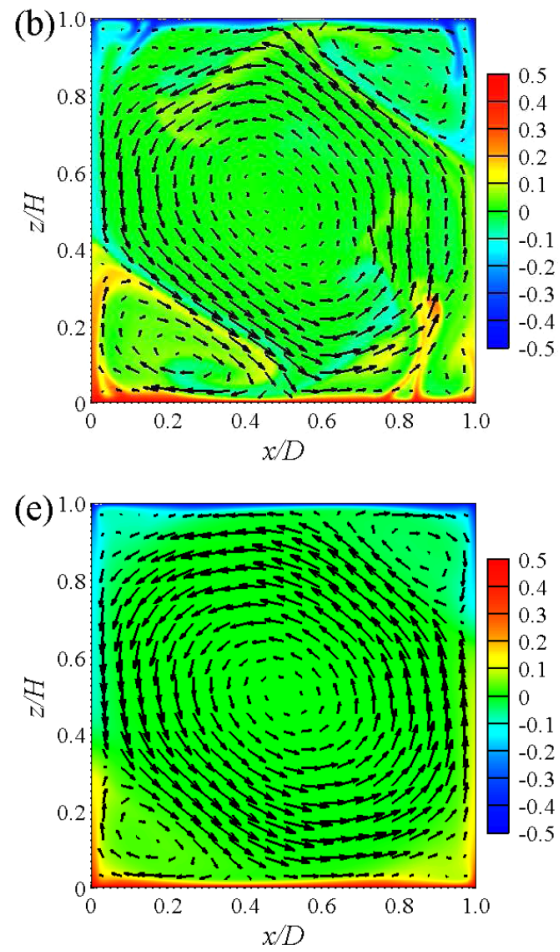
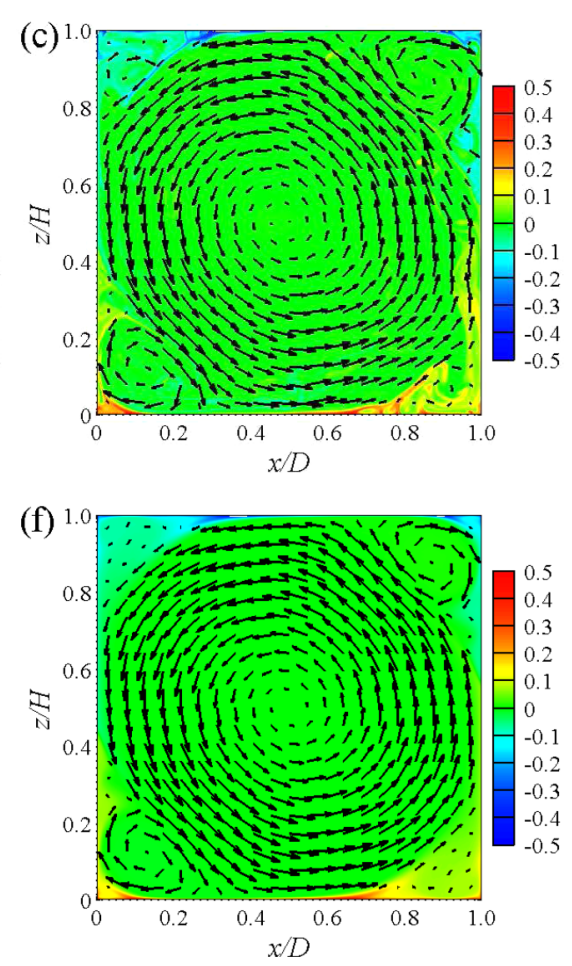

FIG. 2. Instantaneous (a)-(c) and mean (d)-(f) flow fields at three different Rayleigh numbers Ra $=10^{6}$ (left panel), $10^{8}$ (middle panel), and $10^{10}$ (right panel). The magnitudes of velocity and temperature are coded in arrows and color, respectively. 
compute the mean flow fields; cf. Zhou et al. [30] for more details. After this transformation, the direction of the LSC is anticlockwise for all times, and all the statistics are calculated using this method in the remainder of this paper. The corresponding conditional time-averaged flow fields are shown in Figs. 2(d)-2(f). The instantaneous and mean flow fields all show the process by which the LSC evolves from a tilted and nearly elliptical shape at lower Ra to a squarish shape at higher Ra, which is similar to the observation in the 3D situation [46]. However, it should be noted that both the instantaneous and averaged flow fields presented here cannot represent the pure state or typical shape of the LSC as the flow reversals are always accompanied by the growth of the corner rolls, which is bigger for lower Ra. It is further seen that the instantaneous and time-averaged flow fields are more similar at high $\mathrm{Ra}$, thus the wind may have reached a "stable" state with "damped" turbulent fluctuations [7]. It should be noted that the size of the corner flow becomes smaller as Ra increases, which would cause a weaker influence on flow dynamics, as we will see later.

\section{B. Statistics of turbulent fluctuations}

Figure 3 provides the distribution of rms values of velocity and temperature fluctuations. The top row of panels (a)-(c) shows the horizontal rms velocity $u_{\text {rms }}$, the central row (d)-(f) depicts the vertical rms velocity $v_{\text {rms }}$, and the bottom row (g)-(i) gives the rms temperature $\theta_{\text {rms }}$. The left, middle, and right columns are for $\mathrm{Ra}=10^{6}, 10^{8}$, and $10^{10}$, respectively. As mentioned above, at low Ra thermal plumes extend approximately over the entire cell, and since the plumes are more likely to travel through the bulk region, the measured magnitudes of rms velocity and temperature are large in the bulk. As Ra increases, turbulence is promoted, and the high-magnitude regions are more concentrated due to the stronger plume mixing. This can also be seen from the distribution of turbulent (a)

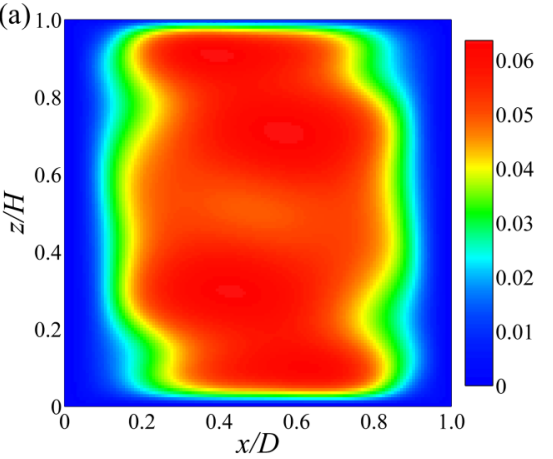

(d) 1
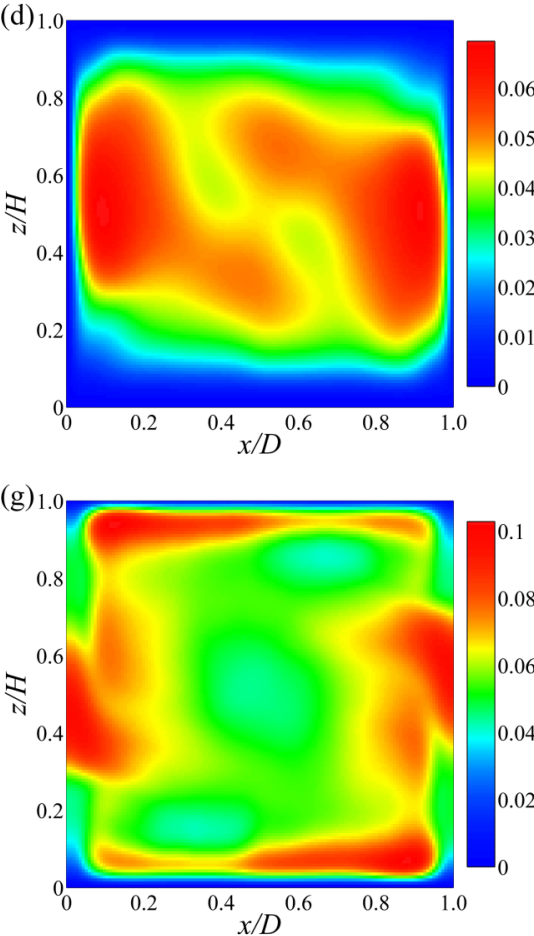
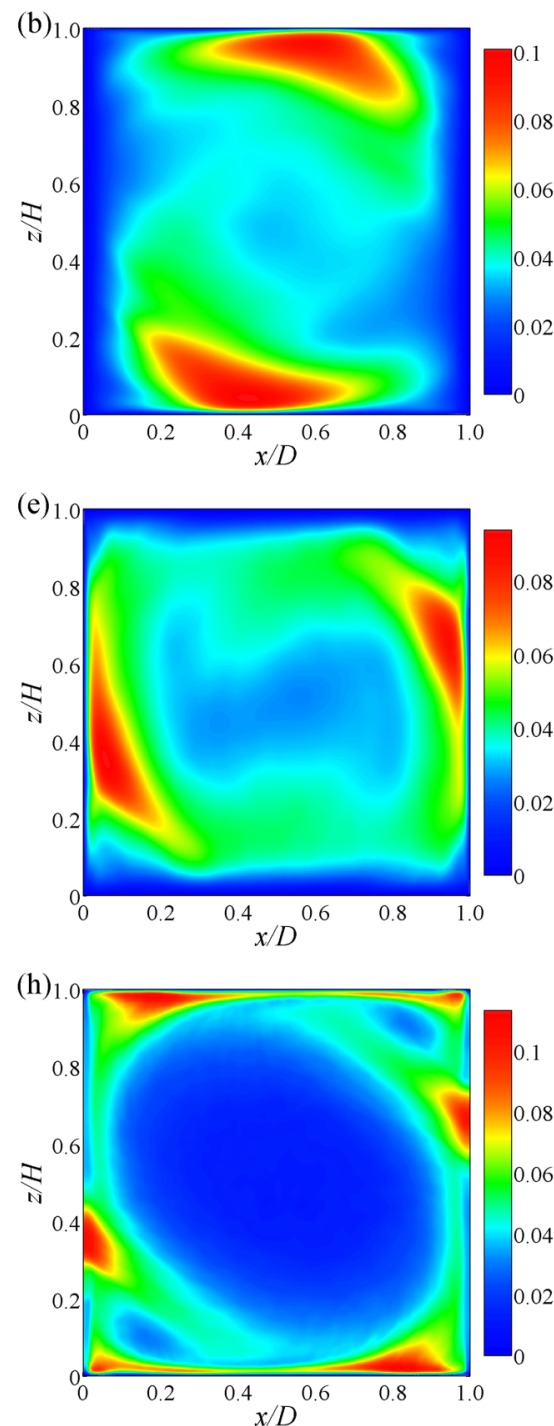
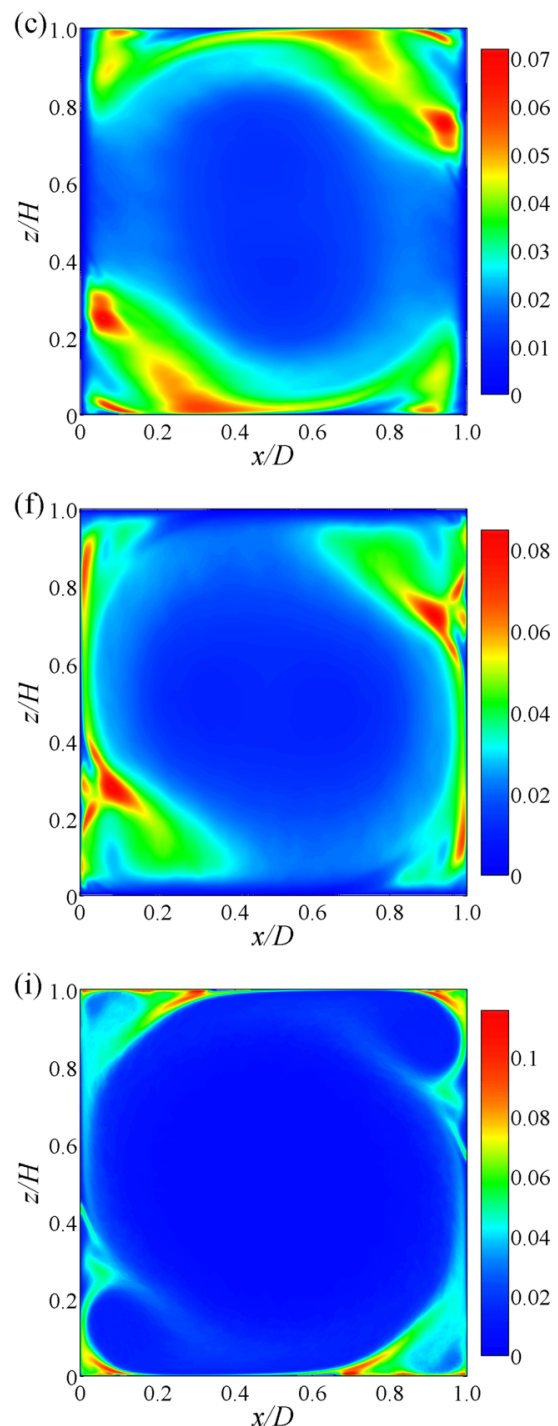

FIG. 3. Root-mean-square values of horizontal velocity fluctuations $u_{\mathrm{rms}}$ (a)-(c), vertical velocity fluctuations $v_{\text {rms }}$ (d)-(f), and temperature fluctuations $\theta_{\text {rms }}(\mathrm{g})-(\mathrm{i})$. Data are obtained at three different Rayleigh numbers (from left to right): $\mathrm{Ra}=10^{6}$ (left panel), $10^{8}$ (middle panel), and $10^{10}$ (right panel). The data used here are the same as those in Figs. 2(d)-2(f). 

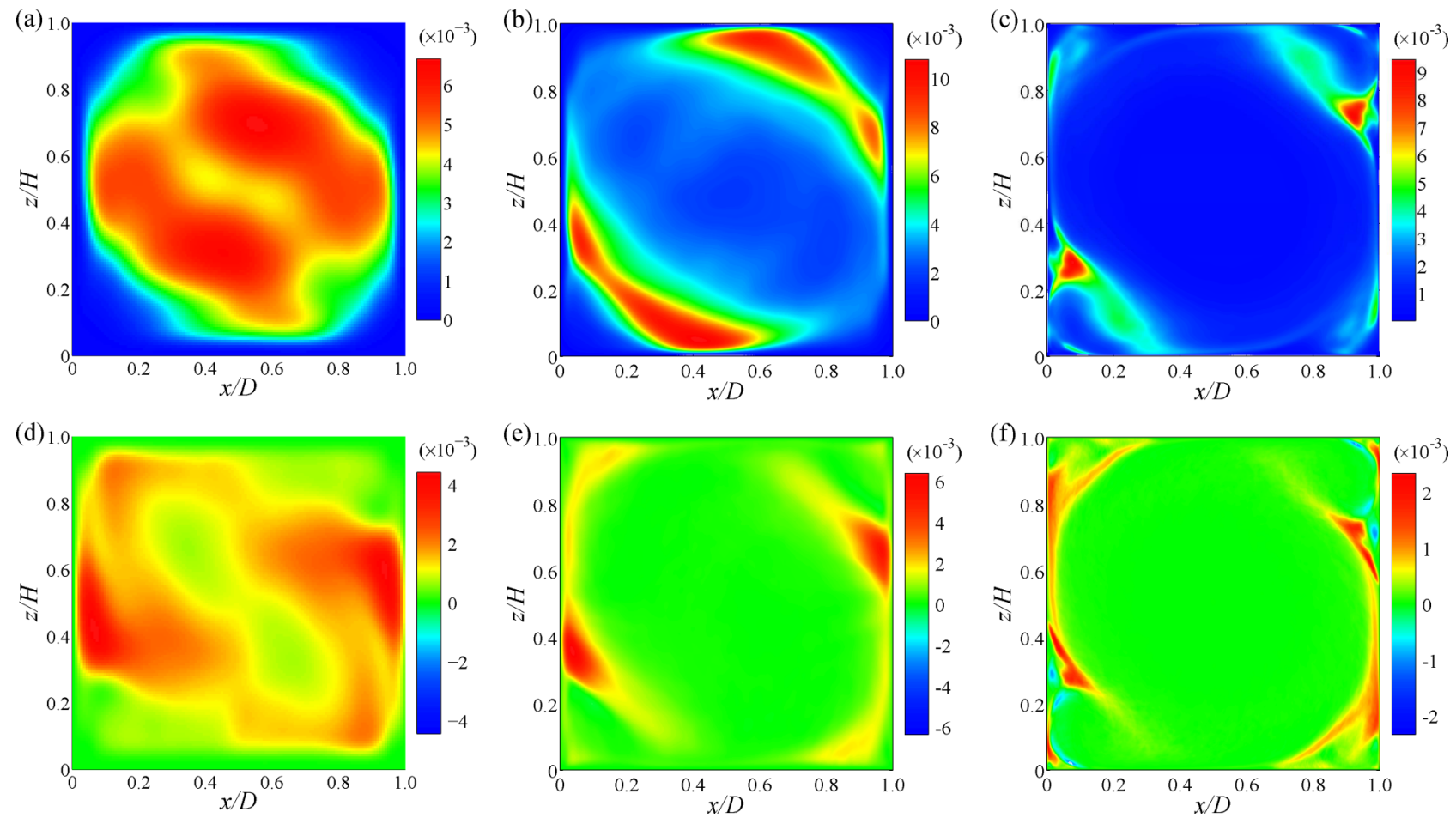

FIG. 4. Turbulent kinetic energy $\left\langle u^{\prime 2}\right\rangle+\left\langle v^{\prime 2}\right\rangle$ (a)-(c) and turbulent heat flux $\left\langle v^{\prime} \theta^{\prime}\right\rangle$ (d)-(f) fields for Ra $=10^{6}$ (left panel), $10^{8}$ (middle panel), and $10^{10}$ (right panel).

kinetic energy $\left\langle u^{\prime 2}\right\rangle+\left\langle v^{\prime 2}\right\rangle$, as shown in Figs. 4(a)-4(c). Here the prime denotes the fluctuation parts of the velocity or temperature field, and the notation $\langle\cdot\rangle$ denotes time average. It has been found that larger fluctuations are closely related to plume clusters [7]. For the present 2D RB system, there are two main reasons for generating high-magnitude fluctuations: one is the plume-ejecting in the lower-right and upper-left corners where the wind-shearing is smaller compared with the central region of the isothermal plates; the other is the competition between the corner-flow rolls and the LSC, which also causes negative values of the local Nusselt number [39]. Figures 4(d)-4(f) plot the color-coded turbulent heat flux $\left\langle v^{\prime} \theta^{\prime}\right\rangle$. Hereafter, it is also referred to as turbulent energy production due to the fluctuation buoyancy force, i.e.,

$$
P_{\theta^{\prime}}=\left\langle v^{\prime} \theta^{\prime}\right\rangle
$$

Simultaneous temperature and velocity measurements in turbulent thermal convection have revealed that high values of heat flux are near the vertical sidewalls, which means that most of the heat is transported by the large-scale convection roll [19]. Similar to the main part of the heat flux $\langle v \theta\rangle$, large amplitudes of turbulent heat flux are associated with the rising and falling plumes as well. This result is also in line with the numerical and experimental Lagrangian measurements in turbulent RB convection [47]. Note that Fig. 4(f) also shows negative values of $\left\langle v^{\prime} \theta^{\prime}\right\rangle$, which correspond to the local countergradient heat transport in 2D thermal convection [39].

The Reynolds stress $-\left\langle u_{i}^{\prime} u_{j}^{\prime}\right\rangle$ represents the mean momentum transport due to turbulent fluctuations. The main components of the Reynolds stress $-\left\langle u^{\prime} v^{\prime}\right\rangle$ for three different $\mathrm{Ra}$ are illustrated in Figs. 5(a)-5(c). As shown in the figures, with increasing Rayleigh number the Reynolds stress is increasingly concentrated near the corners, from which most of the plumes travel. As thermal plumes are coherent structures, their different velocity components naturally have an intense degree of correlation that could result in large Reynolds stress [7]. The description of Krishnamurti and Howard [5] hints that turbulent kinetic energy is transferred from turbulent fluctuations to the mean flow, i.e., the integral of turbulent energy production due to mean shear over the whole flow field is negative [48]; here, the turbulent energy production is given by

$$
P=-\left\langle u_{i}^{\prime} u_{j}^{\prime}\right\rangle \partial\left\langle u_{i}\right\rangle / \partial x_{j}(i, j=1,2) .
$$

However, in most turbulent systems, such as the turbulent flows in channels or pipes, boundary layers, jets, wakes, and mixing layers, the integral value of $P$ is usually positive, and therefore the energy is extracted from the mean motion to increase the turbulent kinetic energy of the flow. The distributions of $P$ for three different values of $\mathrm{Ra}$ are provided in Figs. 5(d)-5(f). It is seen that there are regions with opposite signs of the turbulent energy production, which means that locally the turbulent kinetic energy can be transferred from the mean flow to the turbulent fluctuations and vice versa. It is further found that the integral of $P$ over the whole cell is negative except for the highest Rayleigh number $\mathrm{Ra}=10^{10}$, implying that the mean wind in turbulent thermal convection can be driven by the Reynolds stress, which is different from previous experimental studies [6,7]. According to the turbulent-viscosity hypothesis, the convection flow displays the negative eddy viscosity phenomenon. One possible explanation for this anomalous phenomenon is usually given by analogy with $2 \mathrm{D}$ turbulence, which, under certain conditions, exhibits negative eddy viscosity and other anomalous properties such as "inverse 

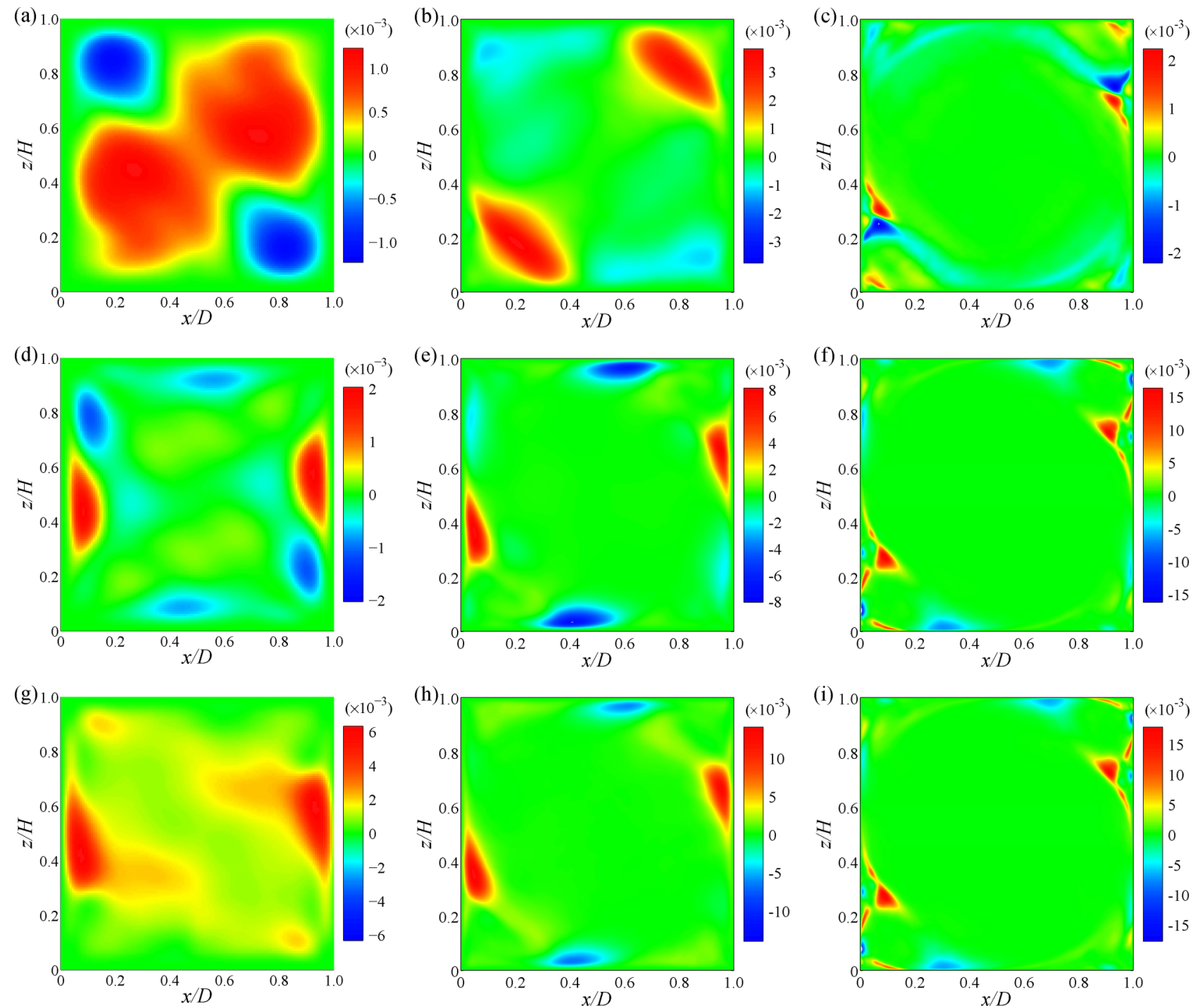

FIG. 5. Main component of the Reynolds stress $-\left\langle u^{\prime} v^{\prime}\right\rangle$ (a)-(c), turbulent energy production due to mean shear $P=-\left\langle u_{i}^{\prime} u_{j}^{\prime}\right\rangle\left\langle u_{i}\right\rangle / \partial x_{j}$ $(i, j=1,2)(\mathrm{d})-(\mathrm{f})$, and total turbulent energy production $P_{0}=P_{\theta^{\prime}}+P$ (g)-(i) for $\mathrm{Ra}=10^{6}$ (left panel), $10^{8}$ (middle panel), and $10^{10}$ (right panel).

energy cascade" [48]. However, why is there a significant transition at $\mathrm{Ra}=10^{10}$ ? To understand this, note that the turbulent production is proportional to the mean shear rate $\partial\left\langle u_{i}\right\rangle / \partial x_{j}$, and both numerical and experimental studies have shown different trends and results of $\partial\left\langle u_{i}\right\rangle / \partial x_{j}$ at higher Ra. On the one hand, the corner-flow rolls play an important role for the large-scale wind reversal in 2D RB convection, and the flow reversals could result in extremely large velocity fluctuations and even negative values of global $\mathrm{Nu}$ [34]. The numerical simulations and experimental investigations all show that for larger Ra, those reversals cannot be observed [24]. At $\mathrm{Ra}=10^{8}$ and $\operatorname{Pr}=0.1$, Burr et al. [6] have shown that the integral of $P$ is positive by solving the two-dimensional Navier-Stokes equations in a square domain. Nevertheless, Sugiyama et al. [24] revealed that for this small $\mathrm{Pr}$, the thermal energy carried by plumes can easily become lost due to large thermal diffusivity, and hence the buildup of the corner roll and the flow reversals is suppressed. Further, our recent study has revealed that for high $\mathrm{Ra}$, thermal mixing is enhanced by the increased turbulent intensity, and then the corner-flow rolls do not have a great influence in the processes of turbulent heat transport for high $\mathrm{Ra}$ [39]. On the other hand, in Fig. 2 we suggest that at $\mathrm{Ra}=10^{10}$ the LSC may have reached an "asymptotic" state with the "damped" turbulent fluctuations, i.e., velocity fluctuations become smaller at $\mathrm{Ra}=$ $10^{10}$, as indicated by the color bar in Figs. 3 and 4 . The two aspects illustrated above mean that the relative strength of the mean shear rate $\partial\left\langle u_{i}\right\rangle / \partial x_{j}$ is larger at $\mathrm{Ra}=10^{10}$, which can contribute to a positive turbulent energy production. In addition, for the system considered here, the temperature field becomes an active scalar, and buoyancy force is responsible for the mean motion of the fluid [49]. The distributions of turbulent energy production due to fluctuating buoyancy force $P_{\theta^{\prime}}$ for the three different Ra are shown in Figs. 4(d)-4(f), and the corresponding maps for total turbulent energy production $P_{0}=P_{\theta^{\prime}}+P$ are depicted in Figs. 5(g)-5(i). The volume averages of $P_{\theta^{\prime}}, P$, and $P_{0}$ at different Ra are presented in Table II. It is shown that although there exist negative values 
TABLE II. Volume average of turbulent energy production due to fluctuating buoyancy force $P_{\theta^{\prime}}$, turbulent energy production due to mean strain $P$, and total turbulent energy production $P_{0}=P_{\theta^{\prime}}+P$ for different Rayleigh numbers (columns from left to right).

\begin{tabular}{lccc}
\hline \hline $\mathrm{Ra}$ & $\left\langle P_{\theta^{\prime}}\right\rangle_{V}\left(\times 10^{3}\right)$ & $\langle P\rangle_{V}\left(\times 10^{3}\right)$ & $\left\langle P_{0}\right\rangle_{V}\left(\times 10^{3}\right)$ \\
\hline $1 \times 10^{6}$ & 1.2780 & -0.0164 & 1.2616 \\
$3 \times 10^{6}$ & 1.3008 & -0.0024 & 1.2984 \\
$1 \times 10^{7}$ & 0.7813 & -0.0484 & 0.7330 \\
$3 \times 10^{7}$ & 0.5237 & -0.0529 & 0.4707 \\
$1 \times 10^{8}$ & 0.5121 & -0.0175 & 0.4946 \\
$3 \times 10^{8}$ & 0.3670 & -0.0039 & 0.3631 \\
$1 \times 10^{9}$ & 0.1887 & -0.0190 & 0.1697 \\
$3 \times 10^{9}$ & 0.1508 & -0.0137 & 0.1370 \\
$1 \times 10^{10}$ & 0.1136 & 0.0218 & 0.1354 \\
\hline \hline
\end{tabular}

of $P_{0}$ in the local regions, the integral of $P_{0}$ is positive over the whole cell. Thus, the contributions to total turbulent energy production are dominated by $P_{\theta^{\prime}}$ in this buoyancy-driven RB convection, and the mean wind is driven both by the Reynolds stress and by the buoyancy of the plumes.

\section{Boundary layer}

We now examine the BL velocity and temperature profiles. Because the velocity and temperature fluctuations are the main subject of the present paper, we focus here mainly on the rms velocity and temperature fields near the conducting plates. In Fig. 6, the profiles of $u_{\text {rms }}$ (a), $v_{\text {rms }}$ (b), and $\theta_{\text {rms }}$ (c) for $\mathrm{Ra}=10^{8}$ are plotted, which have summits close to the plates and then decay as the distance from the plates increases. Here, the profiles are obtained by averaging the rms velocity and temperature fluctuations along the $x$ [Figs. 6(a) and 6(c)] or $z$ direction [Fig. 6(b)], i.e., the profiles of $u_{\mathrm{rms}}, v_{\mathrm{rms}}$, and $\theta_{\mathrm{rms}}$ are either horizontally or vertically averaged. The definition of the corresponding BL thicknesses, i.e., horizontal velocity BL thickness $\delta_{u}$, vertical velocity BL thickness $\delta_{v}$, and temperature BL thickness $\delta_{\theta}$, is also illustrated in the figures using linear fitting of the profiles near the plates, as proposed by Xin and Xia [50]. Figures 7(a)-7(c) present the normalized profiles $u_{\mathrm{rms}} / u_{\mathrm{rms}}^{\max }$ versus $z / \delta_{u}$ (a), $v_{\mathrm{rms}} / v_{\mathrm{rms}}^{\max }$ versus $x / \delta_{v}$ (b), and $\theta_{\mathrm{rms}} / \theta_{\mathrm{rms}}^{\max }$ versus $z / \delta_{\theta}$ (c) at five different values of Ra, where $u_{\mathrm{rms}}^{\max }, v_{\mathrm{rms}}^{\max }$, and $\theta_{\mathrm{rms}}^{\max }$ are the first maximum rms values in the corresponding profiles. It is seen that the shapes of the $v_{\text {rms }}$ and $\theta_{\text {rms }}$ profiles do not significantly change with $\mathrm{Ra}$, whereas some scatter can be observed for the $u_{\text {rms }}$ profiles [see Fig. 7(a)]. Note that the observed scatter is located in the crucial region of the $\mathrm{BL}$ profiles, i.e., the region that can be used to validate different BL theories and where typically the largest deviations for different types of BLs occur. The BL rms profiles exhibit some kind of self-similarity in the present parameter range, indicating that the BL thicknesses defined here are the relevant length scales for the rms velocity (temperature) fields. In addition, when the normalized profiles of $u_{\mathrm{rms}}$ and $v_{\mathrm{rms}}$ are plotted in the same figure, as shown in Figs. 7(d)-7(f), one finds reasonable collapse in the vicinity of plates for all measured Ra.

Figure 8(a) shows the Ra dependence of viscous BL thicknesses normalized by the cell height $H$. The solid circles represent $\delta_{u} / H$ and the upward triangles represent $\delta_{v} / H$. The dashed line marks the best power-law fits, with $\delta_{u} / H \sim$ $\mathrm{Ra}^{-0.26 \pm 0.03}$ and $\delta_{v} / H \sim \mathrm{Ra}^{-0.26 \pm 0.02}$. The result of $\delta_{v} / H$ versus $\mathrm{Ra}$ is different from that of the experimental studies by Qiu and Xia in a cubic RB convection cell in water [16,17], which shows $\delta_{v} / H \sim \mathrm{Ra}^{-0.38 \pm 0.03}$. Figure 8(b) plots the $\mathrm{Re}$ dependence of $\delta_{u} / H$ and $\delta_{v} / H$ with the same symbols, and the best power-law fits yield $\delta_{u} / H \sim \mathrm{Re}^{-0.43 \pm 0.04}$ and $\delta_{v} / H \sim$ $\mathrm{Re}^{-0.43 \pm 0.04}$, which consist of 3D numerical simulations [51]. The results show that the scalings can be affected by the cell geometry and by the Prandtl number. A possible explanation for this could be attributed to the different large-scale flow modes, which are sensitive to the geometry of the convection cell and are of great importance to the dynamics in the BLs [52]. The horizontal and vertical viscous BL thicknesses and their Ra scalings are in line with the scaling exponent -0.22 given by the Grossmann-Lohse (GL) theory, in which the viscous BL thicknesses are defined through the slope criterion of the mean profiles [18,53]. The normalized thermal BL thicknesses $\delta_{\theta} / H$ as a function of $\mathrm{Ra}$ and $\mathrm{Re}$ are, respectively, given in Figs. 8(c) and 8(d). The best power-law fits to the corresponding data give $\delta_{\theta} / H \sim \mathrm{Ra}^{-0.30 \pm 0.01}$ and $\delta_{\theta} / H \sim \mathrm{Re}^{-0.49 \pm 0.01}$. Given the alternative thermal BL thickness defined as $\delta_{\text {th }}=H /(2 \mathrm{Nu})$, the unifying GL theory [18,53] assumes that $\delta_{\text {th }}$ obeys a laminar-like Prandtl-Blasius-Pohlhausen scaling [54]:

$$
\delta_{\text {th }} / H \sim \operatorname{Re}^{-1 / 2} f(\operatorname{Pr}) .
$$

Our present results suggest that $\delta_{\theta}$ follows the same scaling behavior as the theoretical prediction for $\delta_{\text {th }}$ but with a different magnitude. We also note that, although the viscous and thermal BL thicknesses are thinner when compared with those obtained using the slope method based on the mean
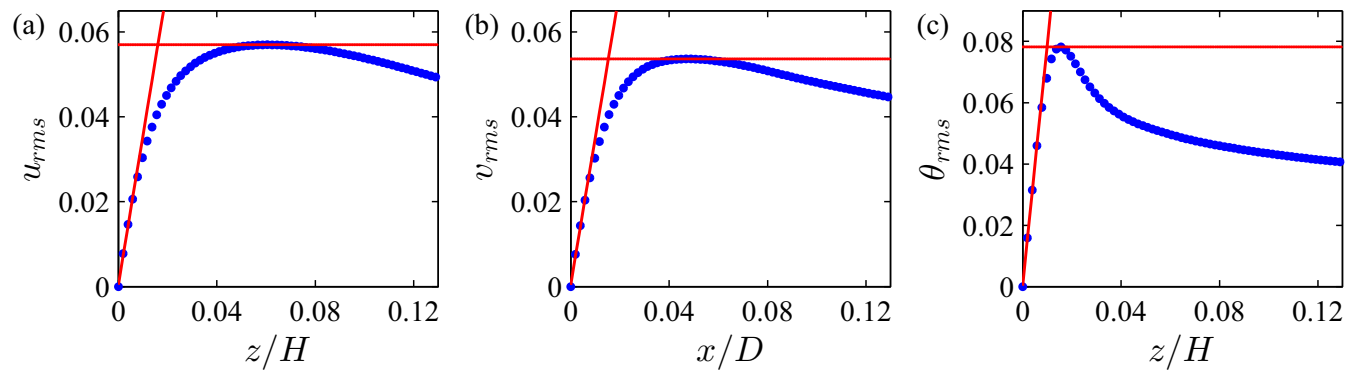

FIG. 6. Profiles of $u_{\mathrm{rms}}$ (a), $v_{\mathrm{rms}}$ (b), and $\theta_{\mathrm{rms}}$ (c) near the plates for Ra $=10^{8}$; the solid lines illustrate the definition of boundary layer thickness. 

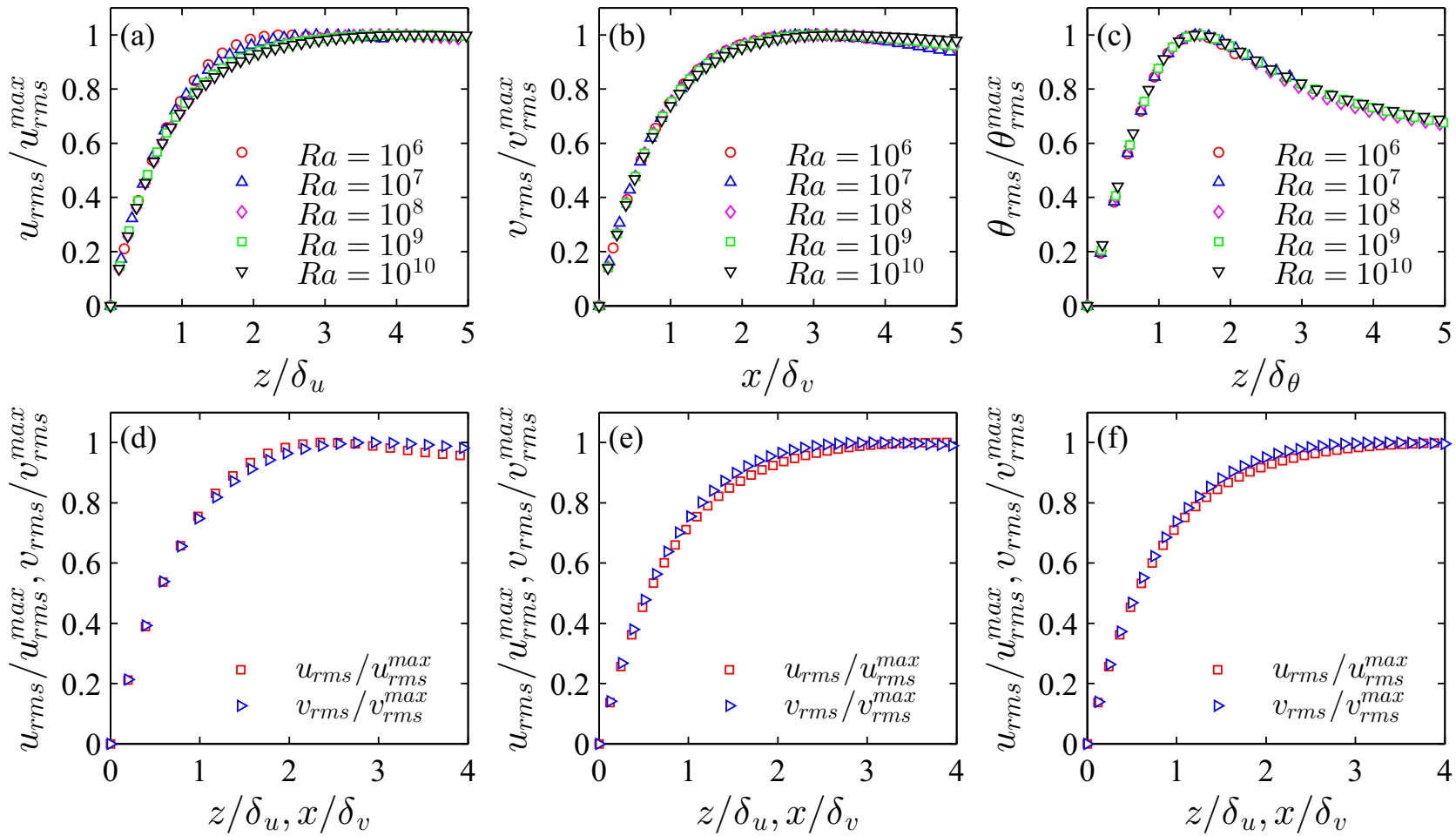

FIG. 7. The normalized profiles for $u_{\mathrm{rms}}(\mathrm{a}), v_{\mathrm{rms}}(\mathrm{b})$, and $\theta_{\mathrm{rms}}$ (c) at five different values of Ra. Normalized profiles compared between $u_{\mathrm{rms}}$ and $v_{\text {rms }}$ for $\mathrm{Ra}=10^{6}(\mathrm{~d}), 10^{8}(\mathrm{e})$, and $10^{10}(\mathrm{f})$.

velocity and temperature profiles, their Ra dependences do not show significant deviation [14,15,51]. In addition, the scaling exponents of $\delta_{v}$ and $\delta_{\theta}$ with respect to Rayleigh and Reynolds numbers do not change in an obvious manner when the BL thicknesses are defined as the distance between the wall and the position at which the rms velocity (temperature) fluctuation reaches its maximum. For reference, Table III summarizes the obtained scaling exponents for the BL thicknesses from several experimental and numerical studies.

Usually, the local Reynolds number is adopted when considering the scaling relationship between the BL thickness and the Rayleigh number Ra [14]. To do this, we calculate the Reynolds numbers, based on the maximum rms velocities, $\operatorname{Re}_{u_{\mathrm{max}}^{\max }}=u_{\mathrm{rms}}^{\max } H / \nu$ and $\operatorname{Re}_{v_{\mathrm{rms}}^{\max }}=v_{\mathrm{rms}}^{\max } H / \nu$, from the rms
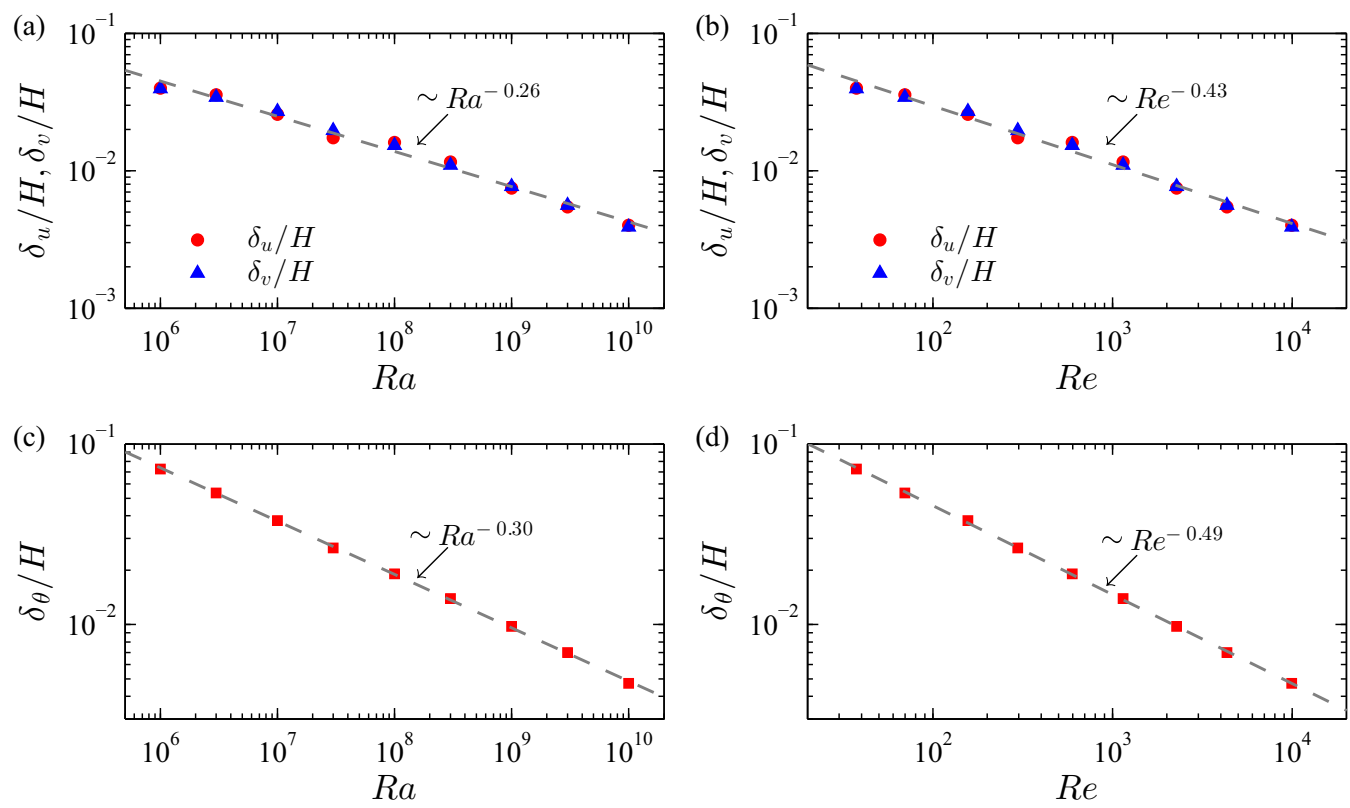

FIG. 8. Ra and Re dependence of $\delta_{u}, \delta_{v}$, and $\delta_{\theta}$. The dashed lines correspond to the least-squares fit to the data and the scaling exponents are summarized in Table III. 
TABLE III. Summary of the fitting results for the normalized viscous and thermal BL thicknesses. The fitting parameters are defined through the power laws: $\delta_{u} / H \sim \mathrm{Ra}^{\gamma_{u}(\mathrm{Ra})}, \delta_{u} / H \sim \mathrm{Re}^{\gamma_{u}(\mathrm{Re})}, \delta_{v} / H \sim \mathrm{Ra}^{\gamma_{v}(\mathrm{Ra})}, \delta_{v} / H \sim \mathrm{Re}^{\gamma_{v}(\mathrm{Re})}, \delta_{\theta} / H \sim \mathrm{Ra}^{\gamma_{\theta}(\mathrm{Ra})}$, and $\delta_{\theta} / H \sim \mathrm{Ra}^{\gamma_{\theta}(\mathrm{Re})}$. The control parameters and cell geometry are also listed. The sources are as follows: (a) Xin and Xia [50], (b) Qiu and Xia [16,17], (c) Sun et al. [14], (d) Zhou and Xia [15], (e) Scheel et al. [51], and (f) the present work.

\begin{tabular}{|c|c|c|c|c|c|c|c|c|c|c|}
\hline Source & $\mathrm{Ra}$ & $\operatorname{Pr}$ & $\Gamma$ & Geometry & $\gamma_{u}(\mathrm{Ra})$ & $\gamma_{u}(\mathrm{Re})$ & $\gamma_{v}(\mathrm{Ra})$ & $\gamma_{v}(\operatorname{Re})$ & $\gamma_{\theta}(\mathrm{Ra})$ & $\gamma_{\theta}(\mathrm{Re})$ \\
\hline $\mathrm{a}$ & $10^{7}-10^{11}$ & $\sim 7$ & $0.5-4.4$ & Cylindrical (3D) & -0.25 & & & & & \\
\hline $\mathrm{b}$ & $10^{8}-10^{10}$ & $\sim 7$ & 1 & Cubic (3D) & -0.25 & & -0.38 & & & \\
\hline $\mathrm{c}$ & $10^{9}-10^{10}$ & 4.3 & 1 & Rectangular (3D) & -0.37 & & & & & \\
\hline $\mathrm{d}$ & $10^{10}-10^{12}$ & 4.3 & 1 & Rectangular (3D) & & & & & -0.31 & \\
\hline $\mathrm{e}$ & $10^{5}-10^{9}$ & 0.7 & 1 & Cylindrical (3D) & -0.23 & -0.45 & & & -0.27 & \\
\hline $\mathrm{f}$ & $10^{6}-10^{10}$ & 5.3 & 1 & Square (2D) & -0.26 & -0.43 & -0.26 & -0.43 & -0.30 & -0.49 \\
\hline
\end{tabular}

profiles. Figure 9(a) shows the $\mathrm{Ra}$ dependence of $\mathrm{Re}_{u_{\mathrm{rms}}^{\max }}$ and $\operatorname{Re}_{v_{\mathrm{rms}}^{\max }}$; the leftward triangles represent $\operatorname{Re}_{u_{\mathrm{rms}}^{\max }}$ and the diamonds represent $\operatorname{Re}_{v_{\mathrm{rms}}^{\max }}$. The best power-law fits to the corresponding data give $\operatorname{Re}_{u_{\mathrm{rms}}^{\max }} \sim \mathrm{Ra}^{0.47 \pm 0.06}$ and $\operatorname{Re}_{v_{\mathrm{rms}}^{\max }} \sim$ $\mathrm{Ra}^{0.48 \pm 0.05}$. For reference, the dashed line with a slope of 0.5 is plotted in Fig. 9(a), as suggested by Xia and his co-workers $[14,16,17,50]$. The viscous BL thicknesses $\delta_{u} / H$ and $\delta_{v} / H$ versus $\mathrm{Re}_{u_{\mathrm{rms}}^{\max }}$ and $\mathrm{Re}_{v_{\mathrm{rms}}^{\max }}$ are provided in log-log plots of Figs. 9(b) and 9(c), respectively. The best power-law fits to the numerical data yield $\delta_{u} / H \sim \operatorname{Re}_{u_{\mathrm{rms}}^{\max }}^{-0.52 \pm 0.11}$ and $\delta_{v} / H \sim$ $\operatorname{Re}_{v_{\mathrm{rms}}^{\max }}^{-0.53 \pm 0.08}$, which also suggests a Prandtl-Blasius-like scaling from the rms perspective for the present flow fields.

\section{Turbulent dissipation rates in the boundary layers}

The kinetic $\varepsilon_{u}$ and thermal $\varepsilon_{\theta}$ energy dissipation rates are of significance in turbulent RB convection: they are closely related to heat-transport processes in this system, and the two exact relations between dissipation rates and Nusselt number are the backbone of the popular GL theory as well
$[1,18,53]$. There have been many experimental $[55,56]$ and numerical [57-60] studies focusing on the statistical properties of dissipation rates in $3 \mathrm{D}$ turbulent $\mathrm{RB}$ convection. In addition, the statistics of $\varepsilon_{u}$ and $\varepsilon_{\theta}$ are comprehensively studied in 2D turbulent thermal convection for different $\mathrm{Pr}$ and $\mathrm{Ra}$ in our former work [44]. In this work, we intend to investigate PDFs of the turbulent kinetic $\varepsilon_{u^{\prime}}$ and thermal $\varepsilon_{\theta^{\prime}}$ energy dissipation rates. The definitions of the two quantities are given by

$$
\varepsilon_{u^{\prime}}(x, z, t)=\frac{1}{2} v \sum_{i, j}\left[\frac{\partial u_{i}^{\prime}(x, z, t)}{\partial x_{j}}+\frac{\partial u_{j}^{\prime}(x, z, t)}{\partial x_{i}}\right]^{2}
$$

and

$$
\varepsilon_{\theta^{\prime}}(x, z, t)=\kappa \sum_{i}\left[\frac{\partial \theta^{\prime}(x, z, t)}{\partial x_{i}}\right]^{2} .
$$

Apart from their close relation to the heat transport, $\varepsilon_{u^{\prime}}$ and $\varepsilon_{\theta^{\prime}}$ are also considered to play a substantial role in determining the statistical characteristics of local velocity and temperature fluctuations. The turbulent thermal dissipation rate $\varepsilon_{\theta^{\prime}}$ was
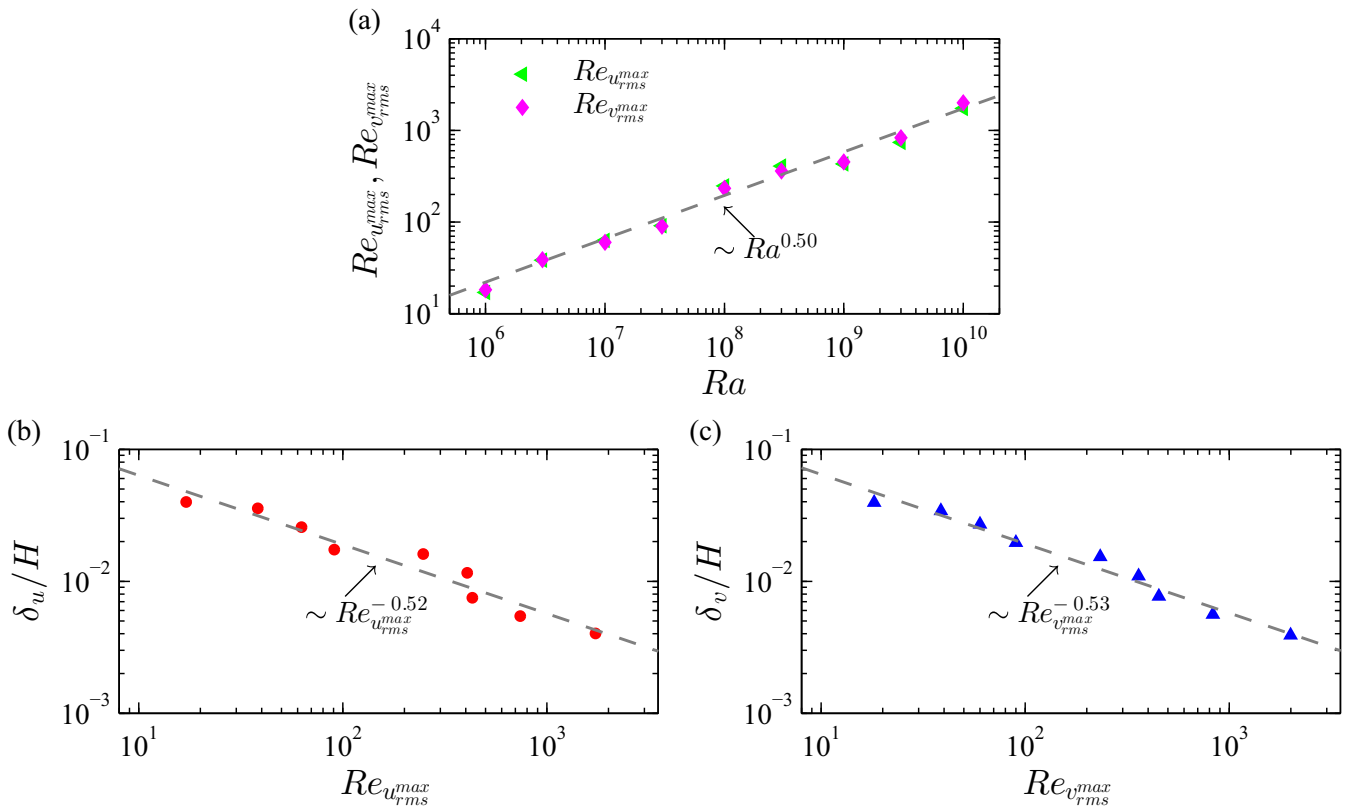

FIG. 9. (a) The rms-velocity-based Reynolds numbers as a function of Ra. The dashed line has a slope of 0.5. (b) The dependence of $\delta_{u} / H$ on $\operatorname{Re}_{u_{\mathrm{rms}}^{\max }}$ and (c) the dependence of $\delta_{v} / H$ on $\operatorname{Re}_{v_{\mathrm{max}} \max }$. The dashed lines in (b) and (c) are the power-law fits to the corresponding data, which yield $\delta_{u} / H \sim \operatorname{Re}_{u_{\mathrm{rms}}^{\text {max }}}^{-0.52 \pm 0.11}$ and $\delta_{v} / H \sim \operatorname{Re}_{v_{\mathrm{rms}}^{\text {max }}}^{-0.53 \pm 0.08}$, respectively. 

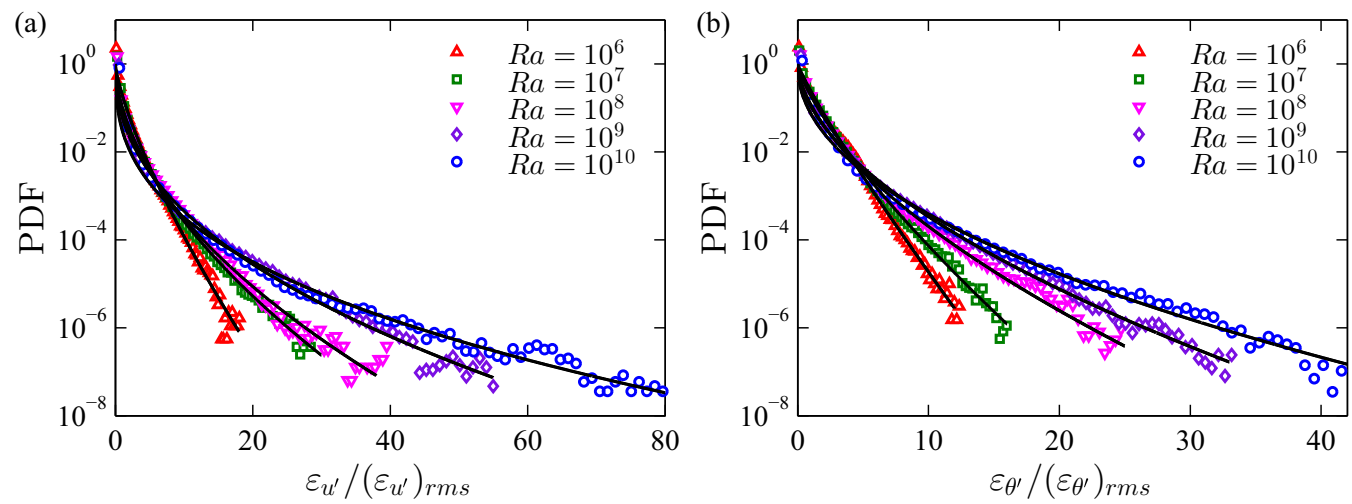

FIG. 10. PDFs of turbulent dissipation rates $\varepsilon_{u^{\prime}}$ (a) and $\varepsilon_{\theta^{\prime}}$ (b) normalized, respectively, by their rms values $\left(\varepsilon_{u^{\prime}}\right)_{\mathrm{rms}}=\sqrt{\left\langle\varepsilon_{u^{\prime}}^{2} \mathrm{BL}, t\right.}$ and $\left(\varepsilon_{\theta^{\prime}}\right)_{\mathrm{rms}}=$ $\sqrt{\left\langle\varepsilon_{\theta^{\prime}}^{2}\right\rangle_{\mathrm{BL}, t}}$ (here the notation $\langle\cdot\rangle_{\mathrm{BL}, t}$ denotes the average over BL parts and over time) for five different Rayleigh numbers. The solid lines are the best fits of stretched exponentials, as given by Eq. (9). The data are calculated within the respective viscous and thermal BLs and over a sequence of statistically independent snapshots.

first obtained from direct measurements of the temperature gradient vector in a cylindrical cell filled with water by $\mathrm{He}$ et al. [55] and He and Tong [56]. They concluded that $\varepsilon_{\theta^{\prime}}$ is mainly generated by thermal plumes, it dominates in the bulk region of the convection cell, and it decreases with increasing Ra. Following their idea, Emran and Schumacher [59] studied the small-scale statistics of temperature and its derivatives in turbulent thermal convection via the DNS method. Their results showed that the tails of the PDFs of $\varepsilon_{\theta^{\prime}}$ in the bulk region can be depicted by a stretched exponential function and always deviate from Gaussian. Here, we are mainly concerned with the two quantities $\varepsilon_{u^{\prime}}$ and $\varepsilon_{\theta^{\prime}}$ within the viscous and thermal BLs, respectively.

The PDFs of turbulent dissipation rates calculated over at least 1000 snapshots equally sampled in a total of 1000 turnover time at different Ra are presented in Fig. 10, where $\varepsilon_{u^{\prime}}$ and $\varepsilon_{\theta^{\prime}}$ are normalized, respectively, by their rms values $\left(\varepsilon_{u^{\prime}}\right)_{\mathrm{rms}}$ and $\left(\varepsilon_{\theta^{\prime}}\right)_{\mathrm{rms}}$. The PDFs of $\varepsilon_{u^{\prime}}$ and $\varepsilon_{\theta^{\prime}}$ both display longer tails with increasing $\mathrm{Ra}$, indicating more intense dissipation that may be attributed to the plume emissions from the thermal BLs. The tails of the PDFs become more extended with increasing $\mathrm{Ra}$, which is different from the experimental results obtained in a cylindrical convection cell by He et al. [55]. They revealed that the normalized PDFs calculated in the cell center at different $\mathrm{Ra}$ can all be brought into agreement. In correspondence with the cases of kinetic and thermal energy dissipation rates in turbulent flows [55,61], the tails of the measured distribution can be described by a stretched exponential function:

$$
h(\chi) \approx e^{-c \chi^{d}},
$$

where $\chi$ can be $\varepsilon_{u^{\prime}} /\left(\varepsilon_{u^{\prime}}\right)_{\mathrm{rms}}$ or $\varepsilon_{\theta^{\prime}} /\left(\varepsilon_{\theta^{\prime}}\right)_{\mathrm{rms}}$, and the fitting parameters $c$ and $d$ are given in Table IV. This function form was first introduced to describe the tails of passive scalar dissipation in the limit of large Peclet and Prandtl numbers in $1 \mathrm{D}$ and 2D cases [62] and then extended to arbitrary space dimensions [63]. In the present work, we use this function to fit the PDF tails of both $\varepsilon_{u^{\prime}}$ and $\varepsilon_{\theta^{\prime}}$. One sees that the value of $d$ decreases monotonically with increasing $\mathrm{Ra}$ for both $\varepsilon_{u^{\prime}}$ and $\varepsilon_{\theta^{\prime}}$, suggesting an increasing degree of small-scale intermittency within the BLs. This is in agreement with the numerical results obtained in 3D cylindrical cells [59]. Note that our present fitted $d$ for $\varepsilon_{\theta^{\prime}}$ obeys the threshold $d>1 / 3$ analytically derived for a passive scalar in the Navier-Stokes flow $[59,64]$.

For the derivation of the refined similarity hypotheses, Kolmogorov conjectured that the dissipation rates are lognormally distributed, i.e., their logarithmic fields have a Gaussian distribution [65]. In the thermally driven flow considered here, one may expect that the warm and cold plumes emitted from the thermal BLs near the conducting plates make the BL region especially interesting for the investigation of deviations of the statistical properties of turbulent fluctuations. To shed some light on the study of turbulent dissipation in BLs, we adopt log-normal distributions to characterize the differences. Figure 11 illustrates the PDFs of $\ln \left(\varepsilon_{u^{\prime}}\right)$ and $\ln \left(\varepsilon_{\theta^{\prime}}\right)$ where the same data sets have been performed in Fig. 10; the PDFs are normalized using the mean and standard deviation values and plotted on a logarithmic scale. The results for all $\mathrm{Ra}$ have some common features, i.e., they are asymmetric and they have negative skewness compared with log-normal distribution. As Ra increases, the tails of the PDFs skew to a higher standardized variable due to the stronger intermittency of the flow fields. Similar behavior was also observed by $\mathrm{He}$ and Tong [56] in cylindrical convection cells filled with water, and their measurements showed that the PDFs of $\ln \left(\varepsilon_{\theta^{\prime}}\right)$ within the temperature BLs have an intense degree of asymmetry compared with those in the cell center, where the plumes are

TABLE IV. The fitting parameters of Eq. (9).

\begin{tabular}{lcccc}
\hline \hline $\mathrm{Ra}$ & $c\left(\varepsilon_{u^{\prime}}\right)$ & $d\left(\varepsilon_{u^{\prime}}\right)$ & $c\left(\varepsilon_{\theta^{\prime}}\right)$ & $d\left(\varepsilon_{\theta^{\prime}}\right)$ \\
\hline $1 \times 10^{6}$ & 1.622 & 0.7463 & 1.407 & 0.8889 \\
$3 \times 10^{6}$ & 2.133 & 0.5857 & 2.136 & 0.6036 \\
$1 \times 10^{7}$ & 2.265 & 0.5608 & 1.649 & 0.7615 \\
$3 \times 10^{7}$ & 2.202 & 0.5589 & 1.729 & 0.7232 \\
$1 \times 10^{8}$ & 2.424 & 0.5243 & 2.123 & 0.6028 \\
$3 \times 10^{8}$ & 2.494 & 0.4989 & 1.900 & 0.5790 \\
$1 \times 10^{9}$ & 2.778 & 0.4435 & 2.200 & 0.5605 \\
$3 \times 10^{9}$ & 3.347 & 0.3713 & 2.319 & 0.5296 \\
$1 \times 10^{10}$ & 3.468 & 0.3657 & 2.589 & 0.4827 \\
\hline \hline
\end{tabular}



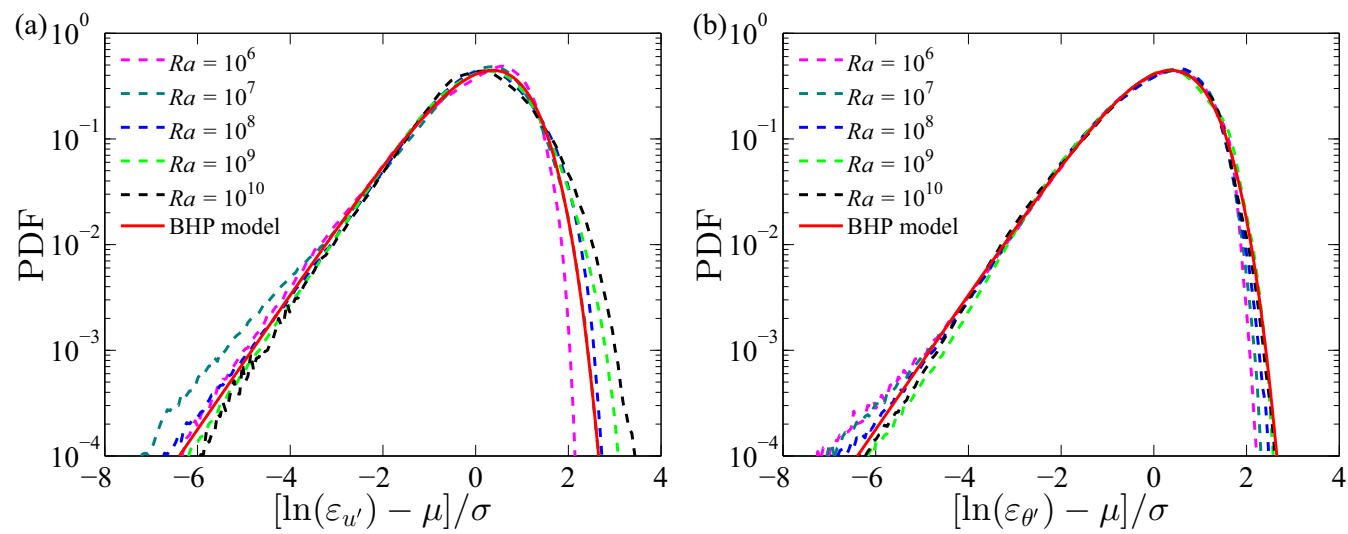

FIG. 11. PDFs of $\left[\ln \left(\varepsilon_{u^{\prime}}\right)-\mu\right] / \sigma$ and $\left[\ln \left(\varepsilon_{\theta^{\prime}}\right)-\mu\right] / \sigma$ for five different values of Ra. Here $\mu$ and $\sigma$ are the mean and standard deviation values of $\ln \left(\varepsilon_{u^{\prime}}\right)$ or $\ln \left(\varepsilon_{\theta^{\prime}}\right)$.

well mixed and the flow is more homogeneous and isotropic. In addition, it is interesting to find that the measured PDFs obey approximately a Bramwell-Holdsworth-Pinton (BHP) distribution, which is

$$
\Pi(y)=K\left(e^{y-e^{y}}\right)^{a}, \quad y=b(w-s),
$$

where the parameters are $a=\pi / 2, b=0.938, s=0.374$, and $K=2.14$. This formula was first introduced to characterize rare fluctuations in a confined turbulent flow and critical phenomena $[66,67]$. The results shown here thus suggest some kind of universal properties of dissipation with respect to different turbulence systems.

\section{SUMMARY AND CONCLUSIONS}

In this paper, we have presented results of various properties of the velocity and temperature fluctuations in $2 \mathrm{D}$ turbulent $\mathrm{RB}$ convection. These were obtained by numerically solving the dimensionless governing equations in vorticity-stream function form in a square domain with $\mathrm{Ra}$ increasing from $10^{6}$ to $10^{10}$ and $\operatorname{Pr}$ fixed at 5.3. The instantaneous and time-averaged flow fields are also investigated, which shows that as $\mathrm{Ra}$ increases, the shape of the LSC becomes more squarelike and the corner flow rolls have a weaker impact on flow dynamics.

It is found that the large amplitudes of velocity and temperature fluctuations are associated with plume emissions and the competition between the LSC and the corner flows. In addition, the present results reveal that turbulent kinetic energy moves in the "opposite" direction, i.e., from fluctuations to the mean flow, implying that the mean wind in turbulent thermal convection can be driven by the Reynolds stress, in an averaged sense. Thus, apart from the buoyancy of thermal plumes, the Reynolds stress is the reason for the generation of the LSC as well, which is different from the conclusions in $3 \mathrm{D}$ experimental studies [6,7]. It should be noted that, although 2D and 3D convection share some similarities, 2D turbulence has its own feature, such as the inverse energy cascade and negative eddy viscosity phenomenon [48], hence the results observed here cannot readily be translated to $3 \mathrm{D}$ convection.

By using the slope method, the boundary layer thicknesses $\left(\delta_{u}, \delta_{v}\right.$, and $\left.\delta_{\theta}\right)$ based on the rms profiles are obtained. The analyses reveals that the shape of the normalized rms profiles depend slightly on Ra and exhibit self-similarity near the plates. More interestingly, the normalized profiles of the rms horizontal velocity near the conducting plates and those of the rms vertical velocity near the sidewalls have a similar shape, and thus they can yield the same thickness for a fixed Ra. In addition, the viscous BL thicknesses $\delta_{u}$ and $\delta_{v}$ both scale as $\sim \mathrm{Ra}^{-0.26 \pm 0.03}$ and $\sim \mathrm{Re}^{-0.43 \pm 0.04}$. Although the thermal BL thicknesses $\delta_{\theta}$ and $\delta_{\text {th }}$ defined by two different ways show different magnitudes, they share similar scalings with respect to $\mathrm{Ra}$ and $\mathrm{Re}$. The Reynolds numbers based on the local maximum rms velocity are also calculated, and the results show a Prandtl-Blasius-like scaling relation.

With regard to turbulent dissipation rates $\varepsilon_{u^{\prime}}$ and $\varepsilon_{\theta^{\prime}}$ within the BL regions, we find that their PDF tails can be well described by a stretched exponential function. These tails become more extended with increasing $\mathrm{Ra}$ due to an increasing degree of small-scale intermittency within the BLs. Furthermore, when the PDFs are plotted on a logarithmic scale, they obey a BHP distribution, which was first introduced to depict rare fluctuations in a confined turbulent flow and critical phenomena [66,67].

\section{ACKNOWLEDGMENTS}

This work was supported by the Natural Science Foundation of China under Grants No. 11572185, No. 11572203, No. 11572221, and No. 11332006, Shanghai Shuguang Project under Grant No. 13SG40, and Program for New Century Excellent Talents in University under Grant No. NCET-13.
[1] G. Ahlers, S. Grossmann, and D. Lohse, Heat transfer and large scale dynamics in turbulent Rayleigh-Bénard convection, Rev. Mod. Phys. 81, 503 (2009).
[2] D. Lohse and K.-Q. Xia, Small-scale properties of turbulent Rayleigh-Bénard convection, Annu. Rev. Fluid Mech. 42, 335 (2010). 
[3] F. Chillà and J. Schumacher, New perspectives in turbulent Rayleigh-Bénard convection, Eur. Phys. J. E 35, 1 (2012).

[4] K.-Q. Xia, Current trends and future directions in turbulent thermal convection, Theor. Appl. Mech. Lett. 3, 052001 (2013).

[5] R. Krishnamurti and L. N. Howard, Large-scale flow generation in turbulent convection, Proc. Natl. Acad. Sci. (USA) 78, 1981 (1981).

[6] U. Burr, W. Kinzelbach, and A. Tsinober, Is the turbulent wind in convective flows driven by fluctuations? Phys. Fluids 15, 2313 (2003).

[7] K.-Q. Xia, C. Sun, and S.-Q. Zhou, Particle image velocimetry measurement of the velocity field in turbulent thermal convection, Phys. Rev. E 68, 066303 (2003).

[8] C. Sun, K. Q. Xia, and P. Tong, Three-dimensional flow structures and dynamics of turbulent thermal convection in a cylindrical cell, Phys. Rev. E 72, 026302 (2005).

[9] K. Petschel, S. Stellmach, M. Wilczek, J. Lülff, and U. Hansen, Dissipation Layers in Rayleigh-Bénard Convection: A Unifying View, Phys. Rev. Lett. 110, 114502 (2013).

[10] K. Petschel, S. Stellmach, M. Wilczek, J. Lülff, and U. Hansen, Kinetic energy transport in Rayleigh-Bénard convection, J. Fluid Mech. 773, 395 (2015).

[11] R. J. Adrian, Variation of temperature and velocity fluctuations in turbulent thermal convection over horizontal surfaces, Int. J. Heat Mass Transf. 39, 2303 (1996).

[12] R. Fernandes and R. Adrian, Scaling of velocity and temperature fluctuations in turbulent thermal convection, Exp. Therm. Fluid Sci. 26, 355 (2002).

[13] R. Du Puits, C. Resagk, A. Tilgner, F. Busse, and A. Thess, Structure of thermal boundary layers in turbulent RayleighBénard convection, J. Fluid Mech. 572, 231 (2007).

[14] C. Sun, Y.-H. Cheung, and K.-Q. Xia, Experimental studies of the viscous boundary layer properties in turbulent RayleighBénard convection, J. Fluid Mech. 605, 79 (2008).

[15] Q. Zhou and K.-Q. Xia, Thermal boundary layer structure in turbulent Rayleigh-Bénard convection in a rectangular cell, J. Fluid Mech. 721, 199 (2013).

[16] X.-L. Qiu and K.-Q. Xia, Spatial structure of the viscous boundary layer in turbulent convection, Phys. Rev. E 58, 5816 (1998).

[17] X.-L. Qiu and K.-Q. Xia, Viscous boundary layers at the sidewall of a convection cell, Phys. Rev. E 58, 486 (1998).

[18] S. Grossmann and D. Lohse, Fluctuations in turbulent RayleighBénard convection: The role of plumes, Phys. Fluids 16, 4462 (2004).

[19] X.-D. Shang, P. Tong, and K.-Q. Xia, Scaling of the Local Convective Heat Flux in Turbulent Rayleigh-Bénard Convection, Phys. Rev. Lett. 100, 244503 (2008).

[20] R. Lakkaraju, R. J. A. M. Stevens, R. Verzicco, S. Grossmann, A. Prosperetti, C. Sun, and D. Lohse, Spatial distribution of heat flux and fluctuations in turbulent Rayleigh-Bénard convection, Phys. Rev. E 86, 056315 (2012).

[21] C. Sun, Q. Zhou, and K.-Q. Xia, Cascades of Velocity and Temperature Fluctuations in Buoyancy-Driven Thermal Turbulence, Phys. Rev. Lett. 97, 144504 (2006).

[22] R. P. J. Kunnen, H. J. H. Clercx, B. J. Geurts, L. J. A. van Bokhoven, R. A. D. Akkermans, and R. Verzicco, Numerical and experimental investigation of structure-function scaling in turbulent Rayleigh-Bénard convection, Phys. Rev. E 77, 016302 (2008).
[23] Q. Zhou and K. Q. Xia, Disentangle plume-induced anisotropy in the velocity field in buoyancy-driven turbulence, J. Fluid Mech. 684, 192 (2011).

[24] K. Sugiyama, R. Ni, R. J. A. M. Stevens, T. S. Chan, S.-Q. Zhou, H.-D. Xi, C. Sun, S. Grossmann, K.-Q. Xia, and D. Lohse, Flow Reversals in Thermally Driven Turbulence, Phys. Rev. Lett. 105, 034503 (2010).

[25] J. Schmalzl, M. Breuer, and U. Hansen, On the validity of twodimensional numerical approaches to time-dependent thermal convection, Europhys. Lett. 67, 390 (2004).

[26] E. P. van der Poel, R. J. Stevens, and D. Lohse, Comparison between two- and three-dimensional Rayleigh-Bénard convection, J. Fluid Mech. 736, 177 (2013).

[27] J. P. Whitehead and C. R. Doering, Ultimate State of TwoDimensional Rayleigh-Bénard Convection Between Free-Slip Fixed-Temperature Boundaries. Phys. Rev. Lett. 106, 244501 (2011).

[28] E. P. van der Poel, R. Ostilla-Mónico, R. Verzicco, S. Grossmann, and D. Lohse, Logarithmic Mean Temperature Profiles and Their Connection to Plume Emissions in Turbulent Rayleigh-Bénard Convection, Phys. Rev. Lett. 115, 154501 (2015).

[29] Q. Zhou, R. J. Stevens, K. Sugiyama, S. Grossmann, D. Lohse, and K.-Q. Xia, Prandtl-Blasius temperature and velocity boundary-layer profiles in turbulent Rayleigh-Bénard convection, J. Fluid Mech. 664, 297 (2010).

[30] Q. Zhou, K. Sugiyama, R. J. Stevens, S. Grossmann, D. Lohse, and K.-Q. Xia, Horizontal structures of velocity and temperature boundary layers in two-dimensional numerical turbulent Rayleigh-Bénard convection, Phys. Fluids 23, 125104 (2011).

[31] E. P. van der Poel, R. J. Stevens, K. Sugiyama, and D. Lohse, Flow states in two-dimensional Rayleigh-Bénard convection as a function of aspect-ratio and Rayleigh number, Phys. Fluids 24, 085104 (2012).

[32] E. P. van der Poel, R. Ostilla-Mónico, R. Verzicco, and D. Lohse, Effect of velocity boundary conditions on the heat transfer and flow topology in two-dimensional Rayleigh-Bénard convection, Phys. Rev. E 90, 013017 (2014).

[33] H. Johnston and C. R. Doering, Comparison of Turbulent Thermal Convection Between Conditions of Constant Temperature and Constant Flux, Phys. Rev. Lett. 102, 064501 (2009).

[34] M. Chandra and M. K. Verma, Flow Reversals in Turbulent Convection Via Vortex Reconnections, Phys. Rev. Lett. 110, 114503 (2013).

[35] B. Podvin and A. Sergent, A large-scale investigation of wind reversal in a square Rayleigh-Bénard cell, J. Fluid Mech. 766, 172 (2015).

[36] S.-N. Xia, Z.-H. Wan, S. Liu, Q. Wang, and D.-J. Sun, Flow reversals in Rayleigh-Bénard convection with non-OberbeckBoussinesq effects, J. Fluid Mech. 798, 628 (2016).

[37] J.-G. Liu, C. Wang, and H. Johnston, A fourth order scheme for incompressible Boussinesq equations, J. Sci. Comput. 18, 253 (2003).

[38] Q. Zhou, Temporal evolution and scaling of mixing in twodimensional Rayleigh-Taylor turbulence, Phys. Fluids 25, 085107 (2013).

[39] Y.-X. Huang and Q. Zhou, Counter-gradient heat transport in two-dimensional turbulent Rayleigh-Bénard convection, J. Fluid Mech. 737, R3 (2013). 
[40] X. Qiu, Y. L. Liu, and Q. Zhou, Local dissipation scales in two-dimensional Rayleigh-Taylor turbulence, Phys. Rev. E 90, 043012 (2014).

[41] Q. Zhou, Y.-X. Huang, Z.-M. Lu, Y.-L. Liu, and R. Ni, Scaleto-scale energy and enstrophy transport in two-dimensional Rayleigh-Taylor turbulence, J. Fluid Mech. 786, 294 (2016).

[42] Q. Zhou and L.-F. Jiang, Kinetic and thermal energy dissipation rates in two-dimensional Rayleigh-Taylor turbulence, Phys. Fluids 28, 045109 (2016).

[43] O. Shishkina, R. J. Stevens, S. Grossmann, and D. Lohse, Boundary layer structure in turbulent thermal convection and its consequences for the required numerical resolution, New $\mathrm{J}$. Phys. 12, 075022 (2010).

[44] Y. Zhang, Q. Zhou, and C. Sun, Statistics of kinetic and thermal energy dissipation rates in two-dimensional turbulent RayleighBénard convection, J. Fluid Mech. 814, 165 (2017).

[45] K. Sugiyama, E. Calzavarini, S. Grossmann, and D. Lohse, Flow organization in two-dimensional non-Oberbeck-Boussinesq Rayleigh-Bénard convection in water, J. Fluid Mech. 637, 105 (2009).

[46] C. Sun and K.-Q. Xia, Scaling of the Reynolds number in turbulent thermal convection, Phys. Rev. E 72, 067302 (2005).

[47] O. Liot, F. Seychelles, F. Zonta, S. Chibbaro, T. Coudarchet, Y. Gasteuil, J. F. Pinton, J. Salort, and F. Chill, Simultaneous temperature and velocity Lagrangian measurements in turbulent thermal convection, J. Fluid Mech. 794, 655 (2016).

[48] A. Tsinober, An Informal Conceptual Introduction to Turbulence (Springer, Berlin, 2009), Vol. 483.

[49] H. D. Xi, S. Lam, and K. Q. Xia, From laminar plumes to organized flows: The onset of large-scale circulation in turbulent thermal convection, J. Fluid Mech. 503, 47 (2004).

[50] Y.-B. Xin and K.-Q. Xia, Boundary layer length scales in convective turbulence, Phys. Rev. E 56, 3010 (1997).

[51] J. Scheel, E. Kim, and K. White, Thermal and viscous boundary layers in turbulent Rayleigh-Bénard convection, J. Fluid Mech. 711, 281 (2012).

[52] S. Grossmann and D. Lohse, On geometry effects in RayleighBénard convection, J. Fluid Mech. 486, 105 (2003).

[53] S. Grossmann and D. Lohse, Scaling in thermal convection: A unifying theory, J. Fluid Mech. 407, 27 (2000).
[54] L. Landau and E. Lifshitz, Fluid Mechanics, 2nd ed. (Pergamon Press, Oxford, 1987).

[55] X. He, P. Tong, and K.-Q. Xia, Measured Thermal Dissipation Field in Turbulent Rayleigh-Bénard Convection, Phys. Rev. Lett. 98, 144501 (2007)

[56] X. He and P. Tong, Measurements of the thermal dissipation field in turbulent Rayleigh-Bénard convection, Phys. Rev. E 79, 026306 (2009)

[57] R. Verzicco and R. Camussi, Numerical experiments on strongly turbulent thermal convection in a slender cylindrical cell, J. Fluid Mech. 477, 19 (2003).

[58] O. Shishkina, Analysis of thermal dissipation rates in turbulent Rayleigh-Bénard convection, J. Fluid Mech. 546, 51 (2005).

[59] M. S. Emran and J. Schumacher, Fine-scale statistics of temperature and its derivatives in convective turbulence, J. Fluid Mech. 611, 13 (2008).

[60] C. S. Ng, A. Ooi, D. Lohse, and D. Chung, Vertical natural convection: Application of the unifying theory of thermal convection, J. Fluid Mech. 764, 349 (2015).

[61] B. W. Zeff, D. D. Lanterman, R. McAllister, R. Roy, E. J. Kostelich, and D. P. Lathrop, Measuring intense rotation and dissipation in turbulent flows, Nature (London) 421, 146 (2003).

[62] M. Chertkov, G. Falkovich, and I. Kolokolov, Intermittent Dissipation of a Passive Scalar in Turbulence, Phys. Rev. Lett. 80, 2121 (1998).

[63] A. Gamba and I. V. Kolokolov, Dissipation statistics of a passive scalar in a multidimensional smooth flow, J. Stat. Phys. 94, 759 (1999).

[64] J. Schumacher and K. R. Sreenivasan, Statistics and geometry of passive scalars in turbulence, Phys. Fluids 17, 125107 (2005).

[65] S. B. Pope, Turbulent Flows (Cambridge University Press, Cambridge, 2000).

[66] S. Bramwell, P. Holdsworth, and J.-F. Pinton, Universality of rare fluctuations in turbulence and critical phenomena, Nature (London) 396, 552 (1998)

[67] S. T. Bramwell, K. Christensen, J.-Y. Fortin, P. C. W. Holdsworth, H. J. Jensen, S. Lise, J. M. López, M. Nicodemi, J. F. Pinton, and M. Sellitto, Universal Fluctuations in Correlated Systems, Phys. Rev. Lett. 84, 3744 (2000). 